\title{
PERIODIC POINT FREE HOMEOMORPHISMS AND IRRATIONAL ROTATION FACTORS
}

\author{
ALEJANDRO KOCSARD
}

\begin{abstract}
We provide a complete characterization of periodic point free homeomorphisms of the 2-torus admitting irrational circle rotations as topological factors. Given a homeomorphism of the 2-torus without periodic points and exhibiting uniformly bounded rotational deviations with respect to a rational direction, we show that annularity and the geometry of its non-wandering set are the only possible obstructions for the existence of an irrational circle rotation as topological factor. Through a very precise study of the dynamics of the induced $\rho$-centralized skew-product, we extend and generalize considerably previous results of Jäger [Jäg09].
\end{abstract}

\section{INTRODUCTION}

The principal aim of this work consists in giving a complete characterization of those homeomorphisms of the two-dimensional torus $\mathbb{T}^{2}$ that admit an irrational circle rotation as topological factor. These topological factors, that will be called $\mathrm{ir}$ rational circle factors for short, are extremely useful in the dynamical study of these homeomorphisms because they determine an invariant cyclic order on the system. In fact, the partition of the torus $\mathbb{T}^{2}$ given by the fibers of the semi-conjugacy map is invariant by the dynamics and all the atoms of the partition have a well-defined rational homological direction, forming an interesting invariant geometric structure that we called torus pseudo-foliation in [KPR18]. Just to mention an application of these irrational circle factors, and the pseudo-foliations that they generate, let us say they played a key role in recent progresses [Koc16, KPS16, PS18] on the so called Franks-Misiurewicz conjecture [FM90, Conjecture].

Regarding necessary conditions for the existence of an irrational circle factor, the first one we encounter is the so called bounded rotational deviations. In fact, if $g: \mathbb{T}=\mathbb{R} / \mathbb{Z} \bigcirc$ is an arbitrary orientation preserving circle homeomorphism and $\tilde{g}: \mathbb{R} \oslash$ is a lift of $g$, its rotation number $\rho(\tilde{g}) \in \mathbb{R}$ can be characterized as the only real number such that

$$
\left|\tilde{g}^{n}(x)-x-n \rho(\tilde{g})\right| \leqslant 1, \quad \forall x \in \mathbb{R}, \forall n \in \mathbb{Z} .
$$

This property implies (see for instance [JT17, Lemma 3.1]) that if $f: \mathbb{T}^{2} ๖$ is an orientation preserving homeomorphism admitting an irrational circle factor, then there exist a rational slope vector $v \in \mathbb{R}^{2} \backslash\{0\}$ and a positive constant $C=$ $C(f, v)>0$ such that for any lift $\tilde{f}: \mathbb{R}^{2} \supset$ of $f$, there is an irrational number $\rho=\rho(\tilde{f}, v) \in \mathbb{R} \backslash \mathbb{Q}$ satisfying

$$
\left|\left\langle\tilde{f}^{n}(z)-z, v\right\rangle-n \rho\right| \leqslant C, \quad \forall z \in \mathbb{R}^{2}, \forall n \in \mathbb{Z} .
$$

So, it is natural to ask whether estimate (1) is also sufficient to guarantee the existence of an irrational circle factor.

It is known that this question has a positive answer in some particular cases. For instance, as a rather straightforward consequence of classical Gottschalk-Hedlund

Date: July 3, 2020.

The autor was partially supported by FAPERJ (Brazil) and CNPq (Brazil). 
theorem [GH55], one can show that any minimal torus homeomorphism verifying (1) necessarily admits an irrational circle factor. A much subtler positive result is due to Jäger [Jäg09, Theorem C], who showed that any area-preserving totally irrational pseudo-rotation of $\mathbb{T}^{2}$ that exhibits uniformly bounded rotational deviations in any direction (i.e. its rotation set reduces to a single point $\tilde{\rho} \in \mathbb{R}^{2}$, where the corresponding rotation on $\mathbb{T}^{2}$ induced by $\tilde{\rho}$ is minimal, and condition (1) holds for every $v \in \mathbb{R}^{2} \backslash\{0\}$ ) is a topological extension of a totally irrational (i.e. minimal) translation of $\mathbb{T}^{2}$, where this semi-conjugacy is constructed as the combination of two "transversal" irrational circle factors. For some time these were the only known positive results. At this point it is interesting to remark that Wang and Zhang have recently proved in [WZ18] the existence of a $C^{\infty}$ area-preserving diffeomorphism of $\mathbb{T}^{2}$ which is a topological extension of a rigid rotation, but it is not conjugate to it.

On the other hand, Jäger and Tal have shown in [JT17, §4] that boundedness of rotational deviations (i.e. estimate (1) holds) does not in general imply the existence of an irrational circle factor for homeomorphisms of the closed annulus $\mathbb{T} \times[0,1]$. Consequently, annularity could be an obstruction for the existence of such factors for homeomorphisms of $\mathbb{T}^{2}$ (see $\$ 2.6$ for precise definitions and details).

In $\S 3$ we will see that the existence of wandering points may be also another obstruction for the existence of irrational circle factors for periodic point free homeomorphisms satisfying (1). In fact, we will see that the topology and geometry of the wandering set play a determinant role in the existence of irrational circle factors (see Definition 2.3 for details).

The main purpose of this work consists in getting a complete characterization of possible obstructions for the existence of such topological factors, showing that annularity and the existence of "large connected components of wandering points" are the only ones. More precisely, our main result is the following

Theorem A. Let $f \in$ Homeo+ $_{+}\left(\mathbb{T}^{2}\right)$ be an orientation preserving, non-eventually annular homeomorphism having small wandering domains. Then, $f$ admits an irrational circle rotation as topological factor if and only if there exists $v \in \mathbb{R}^{2} \backslash\{0\}$ with rational slope so that $f$ exhibits uniformly bounded rotational $v$-deviations, i.e. given any lift $\tilde{f}: \mathbb{R}^{2} ๖$, there exist $\rho \in \mathbb{R} \backslash \mathbb{Q}$ and $C>0$ such that

$$
\left|\left\langle\tilde{f}^{n}(z)-z, v\right\rangle-n \rho\right| \leqslant C, \quad \forall z \in \mathbb{R}^{2}, \forall n \in \mathbb{Z} .
$$

Here small wandering domains means that all connected components of the wandering set are homotopically trivial in $\mathbb{T}^{2}$ and their diameters are eventually small (see Definition 2.3 for details). It is important to remark that Jäger and Tal have proved this result under the area-preserving assumption [JT17, Theorem 1.1].

On the other hand, let us observe that a homeomorphism admitting a periodic point free factor must be periodic point free itself. So this imposes some restrictions to the possible isotopy classes of the torus homeomorphism (see Proposition 2.4 for details).

As consequence of Theorem A, we get the following extension of Jäger's main result of [Jäg09]:

Corollary 1.1. If $f: \mathbb{T}^{2} \bigcirc$ is a totally irrational pseudo-rotation exhibiting uniformly bounded rotational deviations and having small wandering domains (e.g. $f$ is non-wandering), then $f$ is a topological extension of the corresponding minimal rigid translation of $\mathbb{T}^{2}$.

Another consequence of Theorem A is the following

Corollary 1.2. If $f \in \operatorname{Homeo}\left(\mathbb{T}^{2}\right)$ is isotopic to the $k$-Dehn twist $I_{k}$, with $k \neq 0$ (see (12) for definition), and has small wandering domains, then $f$ admits an irrational 
rotation as topological factor if and only if given any a lift $\tilde{f}: \mathbb{R}^{2} ๖$ of $f$, there exist $\rho \in \mathbb{R} \backslash \mathbb{Q}$ and $C>0$ such that

$$
\left|\operatorname{pr}_{2}\left(\tilde{f}^{n}(z)-z\right)-n \rho\right| \leqslant C, \quad \forall z \in \mathbb{R}^{2}, \forall n \in \mathbb{Z},
$$

where $\mathrm{pr}_{2}: \mathbb{R}^{2} \ni(x, y) \mapsto y$.

Let us finish this introduction remarking that putting together our results with some recent ones due to Hauser and Jäger [HJ17], one can completely characterize those periodic point free homeomorphisms of $\mathbb{T}^{2}$ that admits non-trivial Kronecker factors. In fact, a Kronecker system is nothing but a translation on a compact abelian topological group. Its mixed algebro-topological nature endowed the system with a rich variety of structures and allows us to combine different techniques (e.g. metric geometry, character theory) to study its dynamical and ergodic properties. So, it is not surprising at all that the problem of determining the existence of nontrivial Kronecker factors is a very important one in topological and differentiable dynamics.

Since any closed subsystem (i.e. the restriction of the dynamics to a closed invariant subset) of a Kronecker one is (conjugate to a) Kronecker itself, then in general one just considers minimal Kronecker factors and by non-trivial Kronecker system we mean a minimal one which does not reduce to a point (see [Tao09, §2.6] for a very nice exposition about Kronecker systems).

As the reader may be expecting, any non-trivial Kronecker factor of a torus homeomorphism is in fact a minimal torus translation and the semi-conjugacy map cannot increases the dimension. However this result is not completely straightforward and has just been proven by Hauser and Jäger [HJ17] (see Theorem 2.9) in dimension 2 and by Edeko [Ede19] in higher dimensions. So, a non-trivial Kronecker factor of a 2-torus homeomorphism can be either an irrational circle rotation or a totally irrational, i.e. minimal, 2-torus rotation; but the second case can be performed as the combination of two "transversal" circle factors (see $\S 4$ for details), so this is the reason we just focus on the existence of irrational circle factors.

1.1. Strategy of the proofs for the main results. In $\S 4$ we prove Corollaries 1.1 and 1.2 assuming Theorem A.

The proof of the main result of the paper, Theorem A, is performed in $\S \S 5$ and 6. In $\S 5$ we introduce the $\rho$-centralized skew-product, which is a slight modification of similar constructions we performed in [KPR18, Koc16], but adapted to a more general context. In that section we we make a very detailed study of the dynamics of these $\rho$-centralized skew-products, showing the following results: in Proposition 5.4 we prove that an orbit of the original homeomorphism exhibits bounded rotational deviations if and only if the corresponding orbits of the $\rho$-centralized skew-product are bounded. Then, in Theorem 5.5 we characterize the non-wandering set of the $\rho$-centralized skew-product showing, for instance, that this skew-product is nonwandering if and only if the original homeomorphism if non-wandering. Then, in Theorem 5.6 we study the topology of open bounded connected invariant sets of the $\rho$-centralized skew-product showing that, under the hypotheses of Theorem A, their complements have two unbounded connected components on each fiber. Finally, in $\S 6$ we finish the proof of Theorem A, showing that the boundaries of some bounded connected open sets which are invariant under the $\rho$-centralized skew-product can be used to construct the fibers of the semi-conjugacy of the original homeomorphism.

Acknowledgments. The author would like to thank Tobias Jäger and Andrés Koropecki for several useful discussions and to the anonymous referees for their criticisms that helped to improved the readability of the paper. 


\section{Preliminaries And notations}

2.1. General topological dynamics. Throughout this article, $(M, d)$ will denote an arbitrary complete metric space. The (open) ball of radius $r>0$ centered at $x \in M$ will be denoted by $B_{r}(x)$. Given any $A \subset M$, we write $\partial_{X} A$ for the boundary of $A$ in $X$, and $\bar{A}$ for its closure; its diameter is given by

$$
\operatorname{diam} A:=\sup \{d(x, y): x, y \in A\} .
$$

We say $A$ is bounded when $\operatorname{diam} A$ is finite.

If $A$ is connected, we write $\operatorname{cc}(M, A)$ for the connected component of $M$ contain$\operatorname{ing} A$. As usual, we write $\pi_{0}(M)$ to denote the set of connected components of the space $M$.

The group of self-homeomorphisms of $M$ will be denoted by $\operatorname{Homeo}(M)$. We shall write $\operatorname{Homeo}_{0}(M)$ for the subgroup of homeomorphisms of $M$ which are homotopic to the identity.

Given any $f \in \operatorname{Homeo}(M)$, we define its support as the closed set

$$
\text { supp } f:=\overline{\{x \in M: f(x) \neq x\}} \text {. }
$$

When $M$ and $N$ are two topological spaces, we say that $f \in \operatorname{Homeo}(M)$ is a topological extension of $g \in \operatorname{Homeo}(N)$ when there exists a surjective continuous map $h: M \rightarrow N$ such that $h \circ f=g \circ h$. In such a case, we say that $g$ is a topological factor of $f$ and the map $h$ is called a semi-conjugacy. A fiber of the semi-conjugacy is the pre-image by $h$ of any point of $N$. The set of maps that are a semi-conjugacy between $f$ and $g$ will be denoted by $\mathcal{S C}(f, g)$, i.e. it is defined by

$$
\mathcal{S C}(f, g):=\left\{h^{\prime} \in C^{0}(M, N): h^{\prime}(M)=N, h^{\prime} \circ f=g \circ h^{\prime}\right\} .
$$

Whenever $M_{1}, M_{2}, \ldots, M_{n}$ are arbitrary sets, we shall use the generic notation $\mathrm{pr}_{i}: M_{1} \times M_{2} \times \ldots \times M_{n} \rightarrow M_{i}$ to denote the $i^{\text {th }}$-coordinate projection map. Similarly, when $A$ is a subset of $M_{1} \times M_{2} \times \ldots \times M_{n}$ and $x \in M_{1}$ is an arbitrary point, we write $A_{x}$ to denote the "the fiber of $A$ over $x$ " given by

$$
A_{x}:=\left\{\left(x_{2}, \ldots, x_{n}\right) \in M_{2} \times \ldots \times M_{n}:\left(x, x_{2}, \ldots, x_{n}\right) \in A\right\} .
$$

2.1.1. Recurrent and non-wandering points. Let $f \in \operatorname{Homeo}(M)$ be any homeomorphism. A point $x \in M$ is said to be recurrent when there exists an increasing sequence of positive integers $\left(n_{j}\right)_{j \geqslant 1}$ such that $f^{n_{j}}(x) \rightarrow x$, as $j \rightarrow \infty$. An open subset $U \subset M$ is said to be a wandering set (for $f$ ) when $f^{n}(U) \cap U=\varnothing$, for every $n \in \mathbb{Z} \backslash\{0\}$. A point $x \in M$ is called non-wandering when no neighborhood of $x$ is wandering. The non-wandering set of $f$, i.e. the set of non-wandering points, shall be denoted by $\Omega(f)$; its complement, called the wandering set of $f$, will be denoted by $\mathscr{W}(f):=M \backslash \Omega(f)$.

When $M$ is locally connected, each connected component of $\mathscr{W}(f)$ is called a wandering domain. Finally, we say $f$ is non-wandering when $\Omega(f)=M$. We will need a new notion which is stronger than non-wandering:

Definition 2.1. A homeomorphism $f: M ๖$ is said to be $\Omega$-recurrent when for every open subset $U \subset M$ satisfying $U \cap \Omega(f) \neq \varnothing$, there is $n \geqslant 1$ such that

$$
U \cap f^{-n}(U) \cap \Omega(f) \neq \varnothing .
$$

Our main interest in this notion is due to the following elementary

Lemma 2.1. Let $M$ be a complete metric space and $f: M \diamond$ be an $\Omega$-recurrent homeomorphism.

Then, recurrent points are dense within the set $\Omega(f)$. 
Proof. Let $x$ be any non-wandering point and $U$ an arbitrary open neighborhood of $x$. Without loss of generality, we can assume $U$ is bounded in $M$. Then we will inductively defined a sequence of nested open sets $\left\{U_{k}\right\}_{k \geqslant 0}$ and an increasing sequence of positive integers $\left\{n_{k}\right\}_{k \geqslant 0}$ as follows: first, let us define $U_{0}:=U$. Then, assuming $k \geqslant 1$ and $U_{k-1}$ has already been defined, we write

$$
n_{k-1}:=\min \left\{n \geqslant 1: U_{k-1} \cap f^{-n}\left(U_{k-1}\right) \cap \Omega(f) \neq \varnothing\right\} .
$$

Then, we define $U_{k}$ as any open set satisfying the following properties:

$$
\begin{gathered}
\overline{U_{k}} \subset U_{k-1} \cap f^{-n_{k-1}}\left(U_{k-1}\right), \\
U_{k} \cap \Omega(f) \neq \varnothing, \\
\operatorname{diam}\left(U_{k}\right)<\operatorname{diam}\left(U_{k-1}\right) / 2 .
\end{gathered}
$$

Observe that, since $f$ is $\Omega$-recurrent, $U_{k-1}$ is open and $\Omega(f)$ is closed, such an open set $U_{k}$ does exists and the natural number given by $(7)$ is well defined.

By our hypothesis about the diameter of the sets $U_{k}$, it follows there is a unique point $y$ such that

$$
\{y\}=\bigcap_{k=0}^{\infty} U_{k}=\bigcap_{k=0}^{\infty} \overline{U_{k}},
$$

and we claim $y$ is a recurrent point. In fact, $y \in U_{k+1} \subset U_{k} \cap f^{-n_{k}}\left(U_{k}\right)$, for every $k \geqslant 0$, and hence

$$
d\left(f^{n_{k}}(y), y\right) \leqslant \operatorname{diam} U_{k} \rightarrow 0, \quad \text { as } k \rightarrow \infty .
$$

On the other hand, by the very same definition it holds $n_{k} \geqslant n_{k-1}$, for very $k \geqslant 1$. This implies $y$ is a recurrent point and $y \in U$. So, recurrent points are dense among non-wandering points.

2.1.2. Minimal systems. Let $M$ be a compact metric space, $f: M \preceq$ an arbitrary homeomorphism and $K \subset M$ be an $f$-invariant nonempty compact set. We say that $K$ is a minimal set (for $f$ ) when $K$ and the empty set are the only $f$-invariant compact subsets of $K$. When $M$ itself is a minimal set, we say that $f$ is a minimal system or a minimal homeomorphism.

2.1.3. Proximality relations. If $f: M \oslash$ is a homeomorphism of the complete metric space $(M, d)$, we say that two points $x, y \in M$ are $f$-proximal when

$$
\inf \left\{d\left(f^{n}(x), f^{n}(y)\right): n \geqslant 1\right\}=0 .
$$

Analogously we can define the notion of $f^{-1}$-proximality. Notice that, in general, these two notions do not coincide.

2.2. Orbits of open sets. Let $M$ be an arbitrary locally connected complete metric space and $f: M ๖$ be an arbitrary homeomorphism. Given any nonempty connected open set $V$, following Koropecki and Tal [KT14] we define

$$
\mathscr{U}_{f}(V):=\operatorname{cc}\left(\bigcup_{n \in \mathbb{Z}} f^{n}(V), V\right),
$$

where $\operatorname{cc}(\cdot, \cdot)$ denotes the connected component as defined at the beginning of $\S 2.1$. Notice that there exists $N \geqslant 1$ such that $f^{N}\left(\mathscr{U}_{f}(V)\right)=\mathscr{U}_{f}(V)$ if and only if $V$ is not a wandering set. 
2.3. Kronecker factors. Let $(G,+)$ denote an abelian group. For each $a \in G$, let us consider the translation $T_{a} \in \operatorname{Homeo}(G)$ given by $T_{a}: G \ni g \mapsto g+a$. When $(G,+)$ is an abelian compact group, any such map is called a Kronecker system.

Remark 2.2. For the sake of simplicity of notations, given $E \subset G$ and $a \in G$ sometimes we shall write $E+a$ to denote $T_{a}(E)$.

All orbit closures of a Kronecker system are (topologically conjugate to) a Kronecker subsystem themselves. So, there is no significant loss of generality just considering minimal Kronecker systems.

We will say $T_{a}: G ๖$ is a non-trivial Kronecker system when $T_{a}$ is minimal and $G$ is not just a singleton.

By classical arguments one can easily show that any compact abelian group $G$ admits a compatible distance $d_{G}$ which is invariant by any translation, i.e. every Kronecker system is an isometry of $\left(G, d_{G}\right)$. In particular this implies that any Kronecker system is equicontinuous (i.e. the family of its iterates is equicontinuous) when $G$ is endowed with an arbitrary compatible metric.

Reciprocally, it can be shown that any minimal equicontinuous homeomorphism of an arbitrary compact metric space is topologically conjugate to a minimal Kronecker system (see for instance [Tao09, Proposition 2.6.7] for details).

Given a homeomorphism of a compact metric space $f: M ๖$, a Kronecker factor of $f$ will be a Kronecker system $T_{a}: G \circlearrowleft$ which is a topological factor of $f$, i.e. there exists a surjective continuous map $h: M \rightarrow G$ satisfying $h \circ f=T_{a} \circ h$.

We will need the following

Definition 2.2. Let $M$ be a compact metric space and $f: M \diamond$ be a homeomorphism. We say that two points $x, y \in M$ are Kronecker equivalent for $f$ if their images coincides under any semi-conjugacy of a Kronecker factor, i.e. for every Kronecker factor $T_{a}: G ๖$ of $f$ and any $h \in \mathcal{S C}\left(f, T_{a}\right)$, it holds $h(x)=h(y)$ (see (5) for this notation).

On the other hand, we say that $x$ and $y$ are Kronecker separated if and only if $h(x) \neq h(y)$, for every non-trivial Kronecker factor $T_{a}: G \circlearrowleft$ and every $h \in$ $\mathcal{S C}\left(f, T_{a}\right)$.

Remark 2.3. Notice that, if $f: M \bigcirc$ is as in Definition 2.2, then two points $x, y \in M$ which are either $f$-proximal or $f^{-1}$-proximal are necessarily Kronecker equivalent.

2.4. Tori and torus homeomorphisms. We will always consider $\mathbb{R}^{d}$ endowed with the Euclidean inner product $\langle v, w\rangle:=\sum_{i=1}^{d} v_{i} w_{i}$ and the induced Euclidean norm $\|v\|:=\sqrt{\langle v, v\rangle}$.

As usual, the $d$-torus $\mathbb{R}^{d} / \mathbb{Z}^{d}$ will be denoted by $\mathbb{T}^{d}$ and we write $\pi: \mathbb{R}^{d} \rightarrow \mathbb{T}^{d}$ for the canonical quotient projection. Notice we are using the same letter $\pi$ to the denote the torus universal covering map independently of its dimension.

We shall always assume $\mathbb{T}^{d}$ endowed with the distance function $d_{\mathbb{T}^{d}}$ given by

$$
d_{\mathbb{T}^{d}}(x, y):=\min \left\{\|\tilde{x}-\tilde{y}\|: \tilde{x} \in \pi^{-1}(x), \tilde{y} \in \pi^{-1}(y)\right\}, \quad \forall x, y \in \mathbb{T}^{d} .
$$

An open set $D \subset \mathbb{R}^{d}$ is called a fundamental domain for the covering $\pi: \mathbb{R}^{d} \rightarrow \mathbb{T}^{d}$ when $\pi$ is injective on $D$ and $\pi(\bar{D})=\mathbb{T}^{d}$.

As a particular case of Kronecker systems we have torus translations $T_{\alpha}: \mathbb{T}^{d} ๖$, with $\alpha \in \mathbb{T}^{d}$. By some abuse of notation and for the sake of simplicity, if $\alpha \in \mathbb{R}^{d}$, we shall just write $T_{\alpha}$ to denote $T_{\pi(\alpha)}$.

A vector $\alpha \in \mathbb{R}^{d}$ is called rational when $\alpha \in \mathbb{Q}^{d}$; otherwise, it is called irrational. Moreover, it is called totally irrational when the translation $T_{\alpha}: \mathbb{T}^{d} ๖$ is minimal. 
It is well known that given any $f \in \operatorname{Homeo}\left(\mathbb{T}^{d}\right)$, there exists a unique matrix $A_{f} \in \mathrm{GL}(d, \mathbb{Z})$ such that the map

$$
\Delta_{\tilde{f}}:=\tilde{f}-A_{f}: \mathbb{R}^{d} \rightarrow \mathbb{R}^{d}
$$

is $\mathbb{Z}^{d}$-periodic, for any lift $\tilde{f}: \mathbb{R}^{d} ๖$ of $f$; and hence the function $\Delta_{\tilde{f}}$ can be considered as an element of $C^{0}\left(\mathbb{T}^{d}, \mathbb{R}^{d}\right)$. The matrix $A_{f}$ is nothing but a matrix representation of the action induced by $f$ on the first homology group of $\mathbb{T}^{d}$, and one can easily check that two homeomorphisms $f, g \in \operatorname{Homeo}\left(\mathbb{T}^{d}\right)$ are homotopic if and only if $A_{f}=A_{g}$.

Finally, given any $k \in \mathbb{Z}$, we write

$$
I_{k}:=\left(\begin{array}{ll}
1 & k \\
0 & 1
\end{array}\right) \in \operatorname{SL}(2, \mathbb{R})
$$

and define

$$
\operatorname{Homeo}_{k}\left(\mathbb{T}^{2}\right):=\left\{f \in \operatorname{Homeo}\left(\mathbb{T}^{2}\right): A_{f}=I_{k}\right\}
$$

2.4.1. Rotation set and rotation vectors. We write $\mathrm{Homeo}_{0}\left(\mathbb{T}^{d}\right)$ to denote the group of torus homeomorphisms which are homotopic to the identity.

After Misiurewicz and Ziemian [MZ89], given any lift $\tilde{f}: \mathbb{R}^{d} ๖$ of a homeomorphism $f \in \mathrm{Homeo}_{0}\left(\mathbb{T}^{d}\right)$ one defines the rotation set of $\tilde{f}$ by

$$
\rho(\tilde{f})=\bigcap_{m \geqslant 0} \overline{\bigcup_{n \geqslant m}\left\{\frac{\tilde{f}^{n}(z)-z}{n}: z \in \mathbb{R}^{d}\right\}}=\bigcap_{m \geqslant 0} \overline{\bigcup_{n \geqslant m}\left\{\frac{\Delta_{\tilde{f}^{n}}(z)}{n}: z \in \mathbb{T}^{d}\right\}},
$$

where $\Delta_{\tilde{f}}$ is the displacement function given by (11). It can be easily shown that the rotation set $\rho(\tilde{f})$ is always nonempty, compact and connected.

For $d=1$, by classical Poincaré theory [Poi80] of circle homeomorphisms we know that $\rho(\tilde{f})$ is always a singleton and its class modulo $\mathbb{Z}$ depends just on $f$ and not on the chosen lift. So, in such a case one can define $\rho(f):=\pi(\rho(\tilde{f})) \in \mathbb{T}$.

In higher dimensions, in general, the rotation set is not singleton. So, we will say that $f \in \operatorname{Homeo}_{0}\left(\mathbb{T}^{d}\right)$ is a pseudo-rotation whenever $\rho(\tilde{f})$ is a singleton for some, and hence any, lift $\tilde{f}: \mathbb{R}^{d} \supset$ of $f$; and $f$ is said to be a totally irrational pseudo-rotation when $\rho(\tilde{f})$ reduces to a point which is a totally irrational one.

On the other hand, given an $f$-invariant Borel probability measure $\mu$ one can define the $\mu$-rotation vector of $\tilde{f}$ by

$$
\rho_{\mu}(\tilde{f}):=\int_{\mathbb{T}^{d}} \Delta_{\tilde{f}} \mathrm{~d} \mu,
$$

where $\Delta_{\tilde{f}}$ is the displacement function given by (11). By classical convexity arguments and Birkhoff ergodic theorem it can be easily checked that

$$
\operatorname{Conv}(\rho(\tilde{f}))=\left\{\rho_{\mu}(\tilde{f}): \mu \in \mathfrak{M}(f)\right\},
$$

where $\mathfrak{M}(f)$ is the space of $f$-invariant Borel probability measures and $\operatorname{Conv}(\cdot)$ denotes the convex hull operator.

For $d=2$, Misiurewicz and Ziemian [MZ89, Theorem 3.4] showed that the rotation set is convex indeed, so it coincides with the set of rotation vectors of measures.

On the other hand, when $f \in \operatorname{Homeo}_{k}\left(\mathbb{T}^{2}\right)$ with $k \neq 0$, one cannot define the rotation set as above, but at least one can define the vertical rotation set as in 
[Doe97, DM97, AZ05], given by

$$
\rho_{\mathrm{V}}(\tilde{f})=\bigcap_{m \geqslant 0} \overline{\bigcup_{n \geqslant m}\left\{\frac{\operatorname{pr}_{2}\left(\tilde{f}^{n}(z)-z\right)}{n}: z \in \mathbb{R}^{2}\right\}} \subset \mathbb{R},
$$

where $\tilde{f}: \mathbb{R}^{2} \oslash$ is any lift of $f$. Analogously, for any $\mu \in \mathfrak{M}(f)$ one defines its vertical rotation number by

$$
\rho_{\mu, \mathrm{V}}(\tilde{f}):=\int_{\mathbb{T}^{2}} \operatorname{pr}_{2} \circ \Delta_{\tilde{f}} \mathrm{~d} \mu .
$$

2.4.2. Dimension two. Let us fix some especial notations for the two-dimensional case. Given any $v=(a, b) \in \mathbb{R}^{2}$, we define $v^{\perp}:=(-b, a)$. A vector $v \in \mathbb{R}^{2} \backslash\{0\}$ is said to have rational slope when there is $\lambda \in \mathbb{R} \backslash\{0\}$ such that $\lambda v \in \mathbb{Z}^{2}$; and irrational slope otherwise.

We write $\mathbb{A}:=\mathbb{T} \times \mathbb{R}$ for the open annulus. We consider the covering maps $\tilde{\pi}: \mathbb{R}^{2} \rightarrow \mathbb{A}$ and $\hat{\pi}: \mathbb{A} \rightarrow \mathbb{T}^{2}$ given by the natural quotient projections

$$
\begin{aligned}
\tilde{\pi}: \mathbb{R}^{2} \ni(\tilde{x}, \tilde{y}) & \mapsto(\tilde{x}+\mathbb{Z}, \tilde{y}) \in \mathbb{A}, \\
\hat{\pi}: \mathbb{A} \ni(x, \tilde{y}) \mapsto(x, \tilde{y}+\mathbb{Z}) & \in \mathbb{T}^{2} .
\end{aligned}
$$

Observe that $\pi=\hat{\pi} \circ \tilde{\pi}: \mathbb{R}^{2} \rightarrow \mathbb{T}^{2}$.

We will always considered $\mathbb{A}$ endowed with the distance function $d_{\mathbb{A}}$ given by

$$
d_{\mathbb{A}}\left(\hat{z}_{0}, \hat{z}_{1}\right):=\min \left\{\left\|\tilde{z}_{1}-\tilde{z}_{0}\right\|: \tilde{z}_{i} \in \tilde{\pi}^{-1}\left(\hat{z}_{i}\right), i=0,1\right\},
$$

for every $\hat{z}_{i} \in \mathbb{A}$ and $i=0,1$.

For each $s \in \mathbb{R}$, we define $\hat{T}_{s} \in \operatorname{Homeo}_{0}(\mathbb{A})$ by $\hat{T}_{s}:(x, \tilde{y}) \mapsto(x, \tilde{y}+s)$.

If $S$ is any surface, by a topological disk in $S$ we mean an open subset of $S$ which is homeomorphic to the unit disc $\left\{z \in \mathbb{R}^{2}:\|z\|<1\right\}$. Analogously, a topological annulus is an open subset of $S$ which is homeomorphic to $\mathbb{A}$.

If $U \subset \mathbb{T}^{2}$ is an open nonempty set, let us consider the group homomorphism induced by the inclusion on first homology groups $i: H_{1}(U, \mathbb{Z}) \rightarrow H_{1}\left(\mathbb{T}^{2}, \mathbb{Z}\right)$. The set $U$ is said to be inessential when $i=0$, and essential otherwise. The set $U$ is called annular when the image of $i$ has rank 1 and fully essential when its rank is equal to 2 , i.e. when $i$ is surjective.

Analogously, a nonempty open set $U \subset \mathbb{A}$ is said to be inessential when the inclusion morphism $i: H_{1}(U, \mathbb{Z}) \rightarrow H_{1}(\mathbb{A}, \mathbb{Z})$ is identically zero; and it is said to be annular otherwise.

An arbitrary subset $E$ of either $\mathbb{T}^{2}$ or $\mathbb{A}$ is said to be inessential when there exists an inessential open set $U$ containing $E$; otherwise, is said to be essential.

A compact connected subset $C \subset \mathbb{T}^{2}$ is called an annular continuum when its complement $\mathbb{T}^{2} \backslash C$ is homeomorphic to $\mathbb{A}$. On the other hand, we say that a set $C \subset \mathbb{A}$ is an essential annular continuum when it is compact, connected and $\mathbb{A} \backslash C$ has exactly two connected components, which will be denoted by $U^{+}(C)$ and $U^{-}(C)$, and they are characterized by the fact that there is a real number $K>0$ such that $\mathbb{T} \times(K,+\infty) \subset U^{+}(C)$ and $\mathbb{T} \times(-\infty,-K) \subset U^{-}(C)$.

The filling of an inessential open subset $U$ of $\mathbb{T}^{2}$ is defined as the union of $U$ with all the inessential connected components of its complement, and will be denoted by Fill $(U)$. Notice that Fill $(U)$ is an open inessential subset itself.

A connected open set $U \subset \mathbb{T}^{2}(U \subset \mathbb{A}$, respectively) is said to be lift-bounded when every connected component of $\pi^{-1}(U)\left(\tilde{\pi}^{-1}(U)\right.$, respectively) is bounded in $\mathbb{R}^{2}$; and it is called lift-unbounded otherwise. Notice that every lift-bounded set is necessarily inessential, but there do exist open inessential lift-unbounded subsets of $\mathbb{T}^{2}$ and $\mathbb{A}$. 
The main reason why we are interested in the spaces $\operatorname{Homeo}_{k}\left(\mathbb{T}^{2}\right)$ of homeomorphisms given by (13) is given by the following

Proposition 2.4. If $f: \mathbb{T}^{2} \bigcirc$ is an orientation preserving fixed point free homeomorphism, then there exists a unique $k \in \mathbb{Z}$ such that $f$ is topologically conjugate to an element of $\mathrm{Homeo}_{k}\left(\mathbb{T}^{2}\right)$.

Proof. This is a straightforward consequence of Lefschetz fixed point theorem. In fact, if $f$ is orientation preserving and has no fixed point, then it must hold

$$
0=L(f)=\sum_{i=0}^{2}(-1)^{i} \operatorname{tr}\left(f_{\star, i}: H_{i}\left(\mathbb{T}^{2}, \mathbb{Q}\right) \circlearrowleft\right)=2-\operatorname{tr}\left(f_{\star, 1}: H_{1}\left(\mathbb{T}^{2}, \mathbb{Q}\right) \oslash\right),
$$

where $L(f)$ denotes the Lefschetz number of $f$. But the matrix $A_{f} \in \operatorname{SL}(2, \mathbb{Z})$ is a representative of $f_{\star, 1}$, and hence, 1 is the only eigenvalue of the matrix $A_{f}$. If $A_{f}=I_{0}$, i.e. is $A_{f}$ is the identity, we are done; if not, $\operatorname{ker}\left(A_{f}-I_{0}\right)$ is a onedimensional space, and since the matrix $A_{f}-I_{0}$ has integer coefficients, this space is generated by a vector $(a, c) \in \mathbb{Z}^{2}$, with $\operatorname{gcd}(a, c)=1$. So, there exists $b, d \in \mathbb{Z}$ such that $a d-b c=1$ and this implies the matrix

$$
B=\left(\begin{array}{ll}
a & b \\
c & d
\end{array}\right) \in \operatorname{SL}(2, \mathbb{Z}),
$$

and $B^{-1} A_{f} B=I_{k}$, for some $k \in \mathbb{Z}$. Then, the linear map $B$ induces (i.e. is the lift to $\mathbb{R}^{2}$ of) a Lie group automorphism $\bar{B}: \mathbb{T}^{2} ๖$, and one can easily check that $\bar{B}^{-1} \circ f \circ \bar{B} \in \operatorname{Homeo}_{k}\left(\mathbb{T}^{2}\right)$.

Let us recall a classical result about fixed point free plane homeomorphisms due to Brouwer:

Theorem 2.5 (Brouwer's translation theorem, see [Fat87]). Let $f: \mathbb{R}^{2} \circlearrowleft$ be an orientation preserving homeomorphism such that $\operatorname{Fix}(f)=\varnothing$. Then, every $x \in \mathbb{R}^{2}$ is wandering for $f$, i.e. $\Omega(f)=\varnothing$.

If $f \in \operatorname{Homeo}\left(\mathbb{T}^{2}\right)$ and $x \in \Omega(f)$, following Koropecki and Tal [KT14] we say that $x$ is inessential when there exists $\varepsilon>0$ such that the open set $\mathscr{U}_{f}\left(B_{\varepsilon}(x)\right)$, given by (9), is inessential; otherwise is said to be an essential point. Moreover, $x$ is said to be a fully essential point when $\mathscr{U}_{f}\left(B_{\mathcal{\varepsilon}}(x)\right)$ is fully essential, for every $\varepsilon>0$.

We have the following results for periodic point free homeomorphisms:

Proposition 2.6. If $f: \mathbb{T}^{2} ๖$ is a periodic point free homeomorphism and $x \in$ $\Omega(f)$, then $x$ is an essential point.

Proof. This is an easy consequence of Theorem 2.5. In fact, if $x \in \Omega(f)$ and there is an open neighborhood $V$ of $x$ such that $\mathscr{U}_{f}(V)$ is inessential, hence the filling Fill $\left(\mathscr{U}_{f}(V)\right)$, as defined in 2.4.2, is $f^{N}$-invariant, for some $N \in \mathbb{N}$. Notice that Fill $\left(\mathscr{U}_{f}(V)\right)$ is homeomorphic to $\mathbb{R}^{2}$ and $\left.f^{N}\right|_{\text {Fill }\left(\mathscr{U}_{f}(V)\right)}:$ Fill $\left(\mathscr{U}_{f}(V)\right) \circlearrowleft$ exhibits a non-wandering point (because $x \in \Omega(f) \cap V)$. Thus, by Brouwer's translation theorem (Theorem 2.5), $f^{2 N}$ has a fixed point in Fill $\left(\mathscr{U}_{f}(V)\right)$, contradicting the fact that $f$ is periodic point free.

Proposition 2.7. If $f: \mathbb{T}^{2} ๖$ is a periodic point free homeomorphism and $W \subset \mathbb{T}^{2}$ is a lift-bounded wandering domain (i.e. $W$ is a connected component of $\mathscr{W}(f)$ ), then

$$
f^{n}(W) \cap W=\varnothing, \quad \forall n \in \mathbb{Z} \backslash\{0\} .
$$


Proof. Let us suppose there exists $n \in \mathbb{Z} \backslash\{0\}$ such that $f^{n}(W) \cap W \neq \varnothing$. Since $W$ is a connected component of an $f$-invariant set, this implies that $f^{n}(W)=W$. So, if $\tilde{W}$ is a connected component of $\pi^{-1}(W) \subset \mathbb{R}^{2}$, then there exists a homeomorphism $G: \mathbb{R}^{2} \oslash$ which is a lift of $f^{n}$ and such that $G(\tilde{W})=\tilde{W}$. Since $\tilde{W}$ is bounded in $\mathbb{R}^{2}$, this implies that $\Omega(G)$ is nonempty and, invoking Brouwer's translation theorem we conclude that $G^{2}$ has a fixed point, contradicting the fact that $f$ is periodic point free.

Motivated by this result, we propose the following

Definition 2.3. We say that a homeomorphism $f: \mathbb{T}^{2} Ð$ exhibits small wandering domains when every connected component of the wandering set $\mathscr{W}(f)$ is liftbounded and, given any $\delta>0$, there exist just finitely many connected components with diameter larger that $\delta$, i.e. the following set

$$
\left\{W \in \pi_{0}(\mathscr{W}(f)): \operatorname{diam}(W) \geqslant \delta\right\}
$$

is finite, for every $\delta>0$.

From this geometric property of the wandering set we get the following dynamical consequence:

Proposition 2.8. If $f \in \operatorname{Homeo}\left(\mathbb{T}^{2}\right)$ is periodic point free and exhibits small wandering domains, then it is $\Omega$-recurrent (see Definition 2.1).

Proof. Reasoning by contradiction, let us suppose there exists an open set $U \subset \mathbb{T}^{2}$ such that $U \cap \Omega(f) \neq \varnothing$, but

$$
U \cap f^{n}(U) \cap \Omega(f)=\varnothing, \quad \forall n \in \mathbb{Z} \backslash\{0\} .
$$

Let $z$ be an arbitrary point of $U \cap \Omega(f)$ and $\varepsilon$ be a positive number such that the ball of radius $\varepsilon$ centered at $z$ satisfies $\overline{B_{\varepsilon}(z)} \subset U$. Taking into account that $f$ exhibits small wandering domain, we know that

$$
\mathscr{W}_{U}(z, \varepsilon):=\left\{D \in \pi_{0}(\mathscr{W}(f)): B_{\varepsilon}(z) \cap D \neq \varnothing, \bar{D} \cap\left(\mathbb{T}^{2} \backslash U\right) \neq \varnothing\right\}
$$

is a finite set.

Then, let us consider the set

$$
\tau:=\left\{n \in \mathbb{Z} \backslash\{0\}: f^{n}\left(B_{\mathcal{\varepsilon}}(z)\right) \cap B_{\mathcal{\varepsilon}}(z) \neq \varnothing\right\} .
$$

Notice that since $z$ is a non-wandering point, the set $\tau$ is nonempty, and moreover, is infinite.

On the other hand, by (19) we know that

$$
f^{n}\left(B_{\mathcal{\varepsilon}}(z)\right) \cap B_{\mathcal{\varepsilon}}(z) \subset \mathscr{W}(f), \quad \forall n \in \tau .
$$

Then, if $D$ is a connected component of the wandering set $\mathscr{W}(f)$ such that $\bar{D} \subset U$, its boundary $\partial D \subset \Omega(f)$ and hence, by (19), it holds $f^{n}(\bar{D}) \cap U=\varnothing$, for every $n \in \mathbb{Z} \backslash\{0\}$.

This implies that

$$
f^{n}\left(B_{\varepsilon}(z)\right) \cap B_{\varepsilon}(z) \subset \bigcup_{D \in \mathscr{W}_{U}(z, \varepsilon)} D, \quad \forall n \in \tau .
$$

However, since $f$ is periodic point free, by Proposition 2.7 we know that every connected component of $\mathscr{W}(f)$ is indeed a wandering set for $f$, and we know that the set $\mathscr{W}_{U}(z, \varepsilon)$ is finite. So, putting together (20), (21) and (22) we conclude the set $\tau$ is finite, contradicting the fact that $z$ was non-wandering. 
2.5. Kronecker factors of homeomorphisms of $\mathbb{T}^{2}$. As we have already mentioned in $\S 2.3$, any minimal equicontinuous homeomorphism of a compact metric space is topologically conjugate to a Kronecker system. So, invoking a recent result due to Hauser and Jäger [HJ17, Theorem B], we have the following

Theorem 2.9. If $f \in \operatorname{Homeo}\left(\mathbb{T}^{2}\right)$ and $T_{a}: G ๖$ is a minimal Kronecker factor of $f$, then the group $G$ is either equal to $\mathbb{T}^{2}, \mathbb{T}$ or the trivial space $\{*\}$.

According to the classification given by Theorem 2.9, any homeomorphism $f \in$ Homeo $\left(\mathbb{T}^{2}\right)$ admitting a minimal Kronecker factor is a topological extension of an irrational circle rotation, i.e. $f$ admits an irrational circle rotation as topological factor, that will be just call irrational circle factors for short.

The following simple lemma imposes some well-known restrictions for the existence of irrational circle factors:

Lemma 2.10. Let $f: \mathbb{T}^{2} \sqsubseteq$ be an orientation preserving homeomorphism admitting an irrational circle factor and $\tilde{f}: \mathbb{R}^{2} \bigcirc$ be a lift of $f$. Then there exist $\rho \in \mathbb{R} \backslash \mathbb{Q}$, $v \in \mathbb{S}^{1}$ with rational slope and $\mathrm{C}>0$ such that

$$
\left|\left\langle\tilde{f}^{n}(z)-z, v\right\rangle-n \rho\right| \leqslant C, \quad \forall z \in \mathbb{R}^{2}, \forall n \in \mathbb{Z} .
$$

Proof. See for instance [JT17, Lemma 3.1].

The direction of the vector $v^{\perp}$, where $v$ is as in Lemma 2.10, is called the homological direction of the circle factor. Notice that, when $f$ is not homotopic to the identity, the homological direction of a circle factor is unique.

2.6. Rotational deviations. Given an orientation preserving homeomorphism $f: \mathbb{T}^{2} \wp$, we say that $f$ exhibits uniformly bounded $v$-deviations, for some $v \in \mathbb{S}^{1}$, whenever there exists a constant $C>0$ such that given any lift $\tilde{f}: \mathbb{R}^{2} \supset$, there is $\rho \in \mathbb{R}$ satisfying

$$
\left|\left\langle\tilde{f}^{n}(z)-z, v\right\rangle-n \rho\right| \leqslant C, \quad \forall z \in \mathbb{R}^{2}, \forall n \in \mathbb{Z} .
$$

Observe that if the vector $v$ has irrational slope, then the homeomorphism $f$ must be isotopic to the identity. However, in this work, as a consequence of Lemma 2.10, we are mainly concerned with the case where $v$ has rational slope. Moreover, since we are dealing with periodic point free homoemorphisms, by Proposition 2.4 we know that, modulo conjugacy with a torus automorphism, we can assume our homeomorphism $f \in \operatorname{Homeo}_{k}\left(\mathbb{T}^{2}\right)$, for some $k \in \mathbb{Z}$, the vector $v=(0,1)$ and the number $\rho$ is the only element of of the vertical rotation set defined in $§ 2.4 .1$. In such a case we will say that $f$ exhibits uniformly bounded vertical deviations.

As a particular case of our previous definition, a homeomorphism $f: \mathbb{T}^{2} \supset$ is said to be annular when there exist a lift $\tilde{f}: \mathbb{R}^{2} \supset$ of $f$, a vector $v \in \mathbb{S}^{1}$ with rational slope and a constant $C>0$ such that

$$
\left|\left\langle\tilde{f}^{n}(z)-z, v\right\rangle\right| \leqslant C, \quad \forall z \in \mathbb{R}^{2}, \forall n \in \mathbb{Z} .
$$

More generally, we say $f$ is eventually annular when there exists $k \in \mathbb{N}$ such that $f^{k}$ is annular.

We will need the following improvement of Proposition 2.6:

Proposition 2.11. If $f \in \operatorname{Homeo}\left(\mathbb{T}^{2}\right)$ is periodic point free and is not eventually annular, then every non-wandering point is fully essential for $f$.

Proof. This is consequence of Proposition 2.6 and the following simple remark: if $x \in \Omega(f)$ is essential but is not fully essential, then there is an open connected neighborhood $V$ of $x$ such that $\mathscr{U}_{f}(V)$ is an annular set and there is $k \in \mathbb{N}$ such that $f^{k}\left(\mathscr{U}_{f}(V)\right)=\mathscr{U}_{f}(V)$. This clearly implies that $f^{k}$ is an annular homeomorphism. 
On the other hand, we will say that the homeomorphism $f \in \operatorname{Homeo}_{k}\left(\mathbb{T}^{2}\right)$ exhibits unbounded horizontal deviations when

$$
\sup \left\{\left|\operatorname{pr}_{1}\left(\tilde{f}^{n}(z)-z\right)-\operatorname{pr}_{1}\left(\tilde{f}^{n}(w)-w\right)\right|: w, z \in \mathbb{R}^{2}, n \in \mathbb{Z}\right\}=\infty .
$$

Notice that any element of $\operatorname{Homeo}_{k}\left(\mathbb{T}^{2}\right)$ exhibits unbounded horizontal deviations, when $k \neq 0$.

We have the following simple

Lemma 2.12. Let $f \in \operatorname{Homeo}_{k}\left(\mathbb{T}^{2}\right)$ and $\tilde{f}: \mathbb{R}^{2} \oslash$ be a lift of $f$. Then the following properties are equivalent:

(1) estimate (24) holds;

(2) we have

$$
\sup \left\{\left|\operatorname{pr}_{1}\left(\tilde{f}^{n}(z)-z\right)-\operatorname{pr}_{1}\left(\tilde{f}^{n}(w)-w\right)\right|: w, z \in \mathbb{R}^{2}, n \in \mathbb{N}\right\}=\infty ;
$$

(3) it holds

$$
\sup \left\{\left|\operatorname{pr}_{1}\left(\tilde{f}^{-n}(z)-z\right)-\operatorname{pr}_{1}\left(\tilde{f}^{-n}(w)-w\right)\right|: w, z \in \mathbb{R}^{2}, n \in \mathbb{N}\right\}=\infty \text {. }
$$

Proof. First observe that given any $z \in \mathbb{R}^{2}$, we have

$$
\tilde{f}^{n}(z)-\tilde{f}^{n}(z+(0,1))=I_{k}^{n}(0,1)=(k n, 1), \quad \forall n \in \mathbb{Z} .
$$

This implies that, when $k \neq 0,(24),(25)$ and (26) hold simultaneously, for every $f \in \operatorname{Homeo}_{k}\left(\mathbb{T}^{2}\right)$ and any lift $\tilde{f}: \mathbb{R}^{2} \bigcirc$.

So let us just consider the case $k=0$. It is clear that condition (24) holds if and only if either (25) or (26) holds. So, it just remains to prove that (25) and (26) are equivalent.

To prove this, it is enough to observe that, if we write $\tilde{f}=I_{0}+\Delta_{\tilde{f}}$, then it holds $\tilde{f}^{-1}=I-\Delta_{\tilde{f}} \circ \tilde{f}$. So we have

$$
\Delta_{\tilde{f}^{-n}}=-\Delta_{\tilde{f}^{n}} \circ \tilde{f}^{n}, \quad \forall n \geqslant 1,
$$

and this clearly shows that (25) holds if and only if (26) holds.

Finally, a homeomorphism $f \in \operatorname{Homeo}_{0}\left(\mathbb{T}^{2}\right)$ is said to be a pseudo-rotation with uniformly bounded rotational deviations when $f$ is a pseudo-rotation and there is a constant $C>0$ such that

$$
\left\|\tilde{f}^{n}(z)-z-n \bar{\rho}\right\| \leqslant C, \quad \forall z \in \mathbb{R}^{2}, \forall n \in \mathbb{Z},
$$

where $\tilde{f}: \mathbb{R}^{2} \supset$ is a lift of $f$ and its rotation set satisfies $\rho(\tilde{f})=\{\bar{\rho}\}$.

Let us finish this section with two lemmas about torus homeomorphisms exhibiting unbounded deviations in certain direction:

Lemma 2.13. Let $f \in \operatorname{Homeo}_{k}\left(\mathbb{T}^{2}\right), \tilde{f}: \mathbb{R}^{2} \supset$ be a lift of $f$ and suppose $f$ exhibits unbounded horizontal rotational deviations, i.e. condition $(24)$ holds. If $x \in \Omega(f)$ is a fully essential point for $f$ and $V$ is a connected neighborhood of $x$, then it holds

$$
\sup _{n \in \mathbb{N}} \operatorname{diam}\left(\operatorname{pr}_{1}\left(\tilde{f}^{n}(\tilde{V})\right)\right)=\infty,
$$

for every connected component $\tilde{V}$ of $\pi^{-1}(V)$.

Proof. Without loss of generality we can assume $V$ is open and lift-bounded. Since $x$ is fully essential, there are two continuous simple closed curves $\alpha_{1}, \alpha_{2}: \mathbb{T} \rightarrow \mathbb{T}^{2}$ that generates the first homology group $H_{1}\left(\mathbb{T}^{2}, \mathbb{Z}\right)$ and such that their images are contained in $\mathscr{U}_{f}(V)$. This implies there exists $C>0$ such that

$$
\operatorname{diam} D<C, \quad \forall D \in \pi_{0}\left(\mathbb{R}^{2} \backslash \pi^{-1}\left(\alpha_{1} \cup \alpha_{2}\right)\right),
$$


where, by some abuse of notation, we are just writing $\alpha_{i}$ to denote the image of the curve $\alpha_{i}$, and $\pi_{0}(\cdot)$ denotes the set of connected components of the corresponding space.

Then, let us consider the open set

$$
Q:=(0,1)^{2} \cup \bigcup\left\{D \in \pi_{0}\left(\mathbb{R}^{2} \backslash \pi^{-1}\left(\alpha_{1} \cup \alpha_{2}\right)\right), D \cap(0,1)^{2} \neq \varnothing\right\} .
$$

Notice that, by the previous estimate, $Q$ is bounded in $\mathbb{R}^{2}$ and its boundary is contained in $\pi^{-1}\left(\alpha_{1} \cup \alpha_{2}\right)$.

So, if $\tilde{V}$ is an arbitrary connected component of $\pi^{-1}(V)$, for each $z \in \partial Q$ there exist $n_{z} \in \mathbb{Z}$ and $\boldsymbol{p}_{z} \in \mathbb{Z}^{2}$ such that

$$
z \in \tilde{f}^{n_{z}}(\tilde{V})+\boldsymbol{p}_{z^{\prime}}
$$

where we are using the notation introduced in Remark 2.2

By compactness of $\partial Q$, there exist finitely many points $z_{1}, \ldots, z_{\ell} \in \partial Q$ such that

$$
\partial Q \subset \bigcup_{i=1}^{\ell} \tilde{f}^{n_{z_{i}}}(\tilde{V})+\boldsymbol{p}_{z_{i}} .
$$

This implies

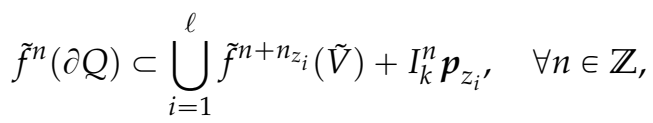

where $I_{k}$ denotes the integer matrix given by (12).

Then, since $Q$ contains a fundamental domain and (24) holds, by (25) of Lemma 2.12 we conclude that

$$
\sup _{n \in \mathbb{N}} \operatorname{diam}\left(\operatorname{pr}_{1}\left(\tilde{f}^{n}(\partial Q)\right)\right)=\infty
$$

Now, for each $n \in \mathbb{Z}, \tilde{f}^{n}(\partial Q)$ is covered by $\ell$ (integer translations of) images of $\tilde{V}$, so estimate (28) must hold.

Lemma 2.14. Let $f \in \operatorname{Homeo}_{k}\left(\mathbb{T}^{2}\right), \tilde{f}: \mathbb{R}^{2} \bigcirc$ be a lift of $f$ and suppose $f$ exhibits uniformly bounded vertical rotational deviations and unbounded horizontal rotational deviations. Let $x \in \Omega(f)$ be a fully essential point for $f, \tilde{V}$ a neighborhood of some point $\tilde{x} \in \pi^{-1}(x) \subset \mathbb{R}^{2}$, and $N_{0}$ be a natural number. Then there exists $m \in \mathbb{N}$, such that for every $j \in\left\{m, m+1, \ldots, m+N_{0}\right\}$, there are $p_{j}, p_{j}^{\prime}, q_{j} \in \mathbb{Z}$, with $p_{j} \neq p_{j}^{\prime}$, such that

$$
\tilde{f}^{j}(\tilde{V}) \cap T_{\left(p_{j}, q_{j}\right)}(\tilde{V}) \neq \varnothing, \text { and } \quad \tilde{f}^{j}(\tilde{V}) \cap T_{\left(p_{j}^{\prime}, q_{j}\right)}(\tilde{V}) \neq \varnothing,
$$

for every $j \in\left\{m, m+1, \ldots, m+N_{0}\right\}$.

Before proving Lemma 2.14, we need the following elementary combinatorial result:

Lemma 2.15. Let $A=\left\{n_{1}, n_{2}, \ldots, n_{\ell}\right\}$ be an arbitrary nonempty set of integer numbers and $N_{0}$ be a natural number. Then, there exists an integer $M_{0} \geqslant N_{0}$ such that for every $m^{\prime} \in \mathbb{N}$ and any function $\xi:\left\{m^{\prime}, m^{\prime}+1, \ldots, m^{\prime}+M_{0}\right\} \rightarrow A$, there is $m \in \mathbb{Z}$ such that

$$
\left\{m, m+1, \ldots, m+N_{0}\right\} \subset\left\{j-\xi(j): j \in\left\{m^{\prime}, m^{\prime}+1, \ldots, m^{\prime}+M_{0}\right\}\right\} .
$$

Proof of Lemma 2.15. Let us suppose the elements of $A$ are ordered in the following way: $n_{1} \leqslant n_{2} \leqslant \ldots \leqslant n_{\ell}$. Then, let us define

$$
\begin{aligned}
M_{0} & :=N_{0}+n_{\ell}-n_{1}, \\
m & :=m^{\prime}-n_{1} .
\end{aligned}
$$


For each $1 \leqslant i \leqslant \ell$, consider the set $B_{i}:=\left\{j-n_{i}: j \in\left\{m^{\prime}, \ldots, m^{\prime}+M_{0}\right\}\right\}$. Then, the lemma easily follows from the following simple remark:

$$
\begin{aligned}
B_{i} \cap B_{k} \supset B_{1} \cap B_{\ell} & =\left\{n \in \mathbb{Z}: m^{\prime}-n_{1} \leqslant n \leqslant m^{\prime}+M_{0}-n_{\ell}\right\} \\
& =\left\{m, m+1, \ldots, m+M_{0}\right\},
\end{aligned}
$$

for every $1 \leqslant i \leqslant k \leqslant \ell$.

Proof. Without loss of generality we can assume $\tilde{V}=B_{r}(\tilde{x})$, with $0<r<1 / 4$. Then, $V:=\pi(\tilde{V})=B_{r}(x) \subset \mathbb{T}^{2}$, where $x=\pi(\tilde{x}) \in \Omega(f)$ is a fully essential point for $f$.

Repeating the argument we used in the proof of Lemma 2.13, there are two continuous simple closed curves $\alpha_{1}, \alpha_{2}: \mathbb{T} \rightarrow \mathbb{T}^{2}$ that generate the first homology group $H_{1}\left(\mathbb{T}^{2}, \mathbb{Z}\right)$ and such that their images are contained in $\mathscr{U}_{f}(V)$.

Then let us consider the fundamental domain $Q$ given by (29). Let $z_{1}, \ldots, z_{\ell} \in$ $\partial Q, n_{z_{1}}, \ldots, n_{z_{\ell}} \in \mathbb{Z}$ and $\boldsymbol{p}_{z_{1}}, \ldots, \boldsymbol{p}_{z_{\ell}} \in \mathbb{Z}^{2}$ such that condition (30) holds. Let $M_{0}$ be the natural number given by Lemma 2.15 associated to the natural numbers $n_{z_{1}}, \ldots, n_{z_{\ell}}$ and $N_{0}$.

Let us consider the set

$$
\check{Q}:=Q \cup \bigcup_{i=1}^{\ell} \tilde{f}^{n_{z_{i}}}(\tilde{V})+\boldsymbol{p}_{z_{i}} .
$$

Now, since $f$ exhibits bounded vertical rotational deviations, we know that its vertical rotation set given by (16) is a singleton and there exists a real constants $C_{0}>0$ such that

$$
\left|\operatorname{pr}_{2}\left(\tilde{f}^{n}(z)-z\right)-n \rho\right| \leqslant C_{0}, \quad \forall z \in \mathbb{R}^{2}, \forall n \in \mathbb{Z},
$$

where $\rho_{\mathrm{V}}(\tilde{f})=\{\rho\}$.

This implies that

$$
\tilde{f}^{n}(\tilde{V}) \subset S_{n}:=\left\{z \in \mathbb{R}^{2}:\left|\operatorname{pr}_{2}(z-\tilde{x})-n \rho\right| \leqslant C_{0}+1 / 4\right\}, \quad \forall n \in \mathbb{Z} .
$$

Then, consider the set of integer numbers

$$
E_{n}:=\left\{q \in \mathbb{Z}: \exists p \in \mathbb{Z}, T_{(p, q)}(\check{Q}) \cap S_{n} \neq \varnothing\right\}, \quad \forall n \in \mathbb{Z} .
$$

Since $\check{Q}$ has finite diameter, we know that

$$
\sharp E_{n} \leqslant 2 C_{0}+\operatorname{diam} \check{Q}+4, \quad \forall n \in \mathbb{Z},
$$

where $\sharp(\cdot)$ denotes the cardinality of the set, and on the other hand it clearly holds

$$
\check{Q} \cap T_{p}(\check{Q})=\varnothing, \quad \forall p \in \mathbb{Z}^{2}, \text { with }\|p\|>\operatorname{diam} \check{Q} \text {. }
$$

Taking into account (35), (36), we consider an integer number $L \in \mathbb{N}$ such that

$$
L>\ell\left(2 C_{0}+\operatorname{diam} \check{Q}+4\right)+1 \text {. }
$$

Now, by Lemma 2.13

$$
\sup _{n \in \mathbb{N}} \operatorname{diam}\left(\operatorname{pr}_{1}\left(\tilde{f}^{n}(\tilde{V})\right)\right)=\infty .
$$

So, we can find a positive integer $m^{\prime}$ such that for every $j \in\left\{m^{\prime}, m^{\prime}+1, \ldots, m^{\prime}+M_{0}\right\}$, there are $\boldsymbol{p}_{1}^{(j)}, \ldots, \boldsymbol{p}_{L}^{(j)} \in \mathbb{Z}^{2}$ with $\left\|\boldsymbol{p}_{r}^{(j)}-\boldsymbol{p}_{s}^{(j)}\right\|>\operatorname{diam} \check{Q}$, for every $1 \leqslant r<s \leqslant L$, and satisfying

$$
\tilde{f}^{j}(\tilde{V}) \cap T_{\boldsymbol{p}_{r}^{(j)}}(Q) \neq \varnothing, \quad \forall j \in\left\{m^{\prime}, \ldots, m^{\prime}+M_{0}\right\}, \forall r \in\{1, \ldots, L\},
$$


where

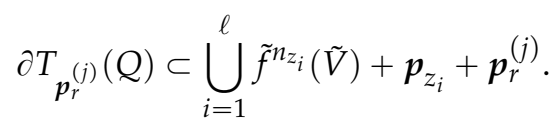

This means that for each $j \in\left\{m^{\prime}, \ldots, m^{\prime}+M_{0}\right\}$ and every $r \in\{1, \ldots, L\}$, there exists $z_{j, r} \in\left\{z_{1}, \ldots, z_{\ell}\right\}$ such that

$$
\tilde{f}^{j}(\tilde{V}) \cap\left(\tilde{f}^{n_{j, r}}(\tilde{V})+\boldsymbol{p}_{z_{j, r}}+\boldsymbol{p}_{r}^{(j)}\right) \neq \varnothing .
$$

Observe that, by $(36)$, the sets $T_{\boldsymbol{p}_{1}^{(j)}}(\check{Q}), T_{\boldsymbol{p}_{2}^{(j)}}(\check{Q}), \ldots, T_{\boldsymbol{p}_{L}^{(j)}}(\check{Q})$ are two-by-two disjoint which implies that

$$
\left(\tilde{f}^{n_{j, r}}(\tilde{V})+\boldsymbol{p}_{z_{j, r}}+\boldsymbol{p}_{r}^{(j)}\right) \cap\left(\tilde{f}^{n_{z_{j, s}}}(\tilde{V})+\boldsymbol{p}_{z_{j, s}}+\boldsymbol{p}_{s}^{(j)}\right)=\varnothing,
$$

for all $j \in\left\{m^{\prime}, \ldots, m^{\prime}+M_{0}\right\}$, and any $1 \leqslant r<s \leqslant L$.

Then, by (33) and (34) we yield

$$
\operatorname{pr}_{2}\left(\boldsymbol{p}_{r}^{(j)}\right) \in E_{j}, \quad \forall r \in\{1, \ldots, L\},
$$

and any $m^{\prime} \leqslant j \leqslant m^{\prime}+M_{0}$. Therefore, putting together this last relation with (35), (38), (39) and (37), we conclude that for each $j \in\left\{m^{\prime}, \ldots, m^{\prime}+M_{0}\right\}$, there are $1 \leqslant r<s \leqslant L$ such that $z_{j, r}=z_{j, s}$ and $\operatorname{pr}_{2}\left(\boldsymbol{p}_{r}^{(j)}\right)=\operatorname{pr}_{2}\left(\boldsymbol{p}_{s}^{(j)}\right)$. So, for each $j$ we get

$$
\begin{aligned}
& \tilde{f}^{j-n_{z_{j, r}}(\tilde{V})} \cap\left(\tilde{V}+I_{k}^{-n_{z_{j, r}}}\left(\boldsymbol{p}_{z_{j, r}}-\boldsymbol{p}_{r}^{(j)}\right)\right) \neq \varnothing, \\
& \tilde{f}^{j-n_{z_{j, s}}(\tilde{V})} \cap\left(\tilde{V}+I_{k}^{-n_{z_{j, s}}}\left(\boldsymbol{p}_{z_{j, s}}-\boldsymbol{p}_{s}^{(j)}\right)\right) \neq \varnothing,
\end{aligned}
$$

where $I_{k}$ is the matrix given by $(12), \boldsymbol{p}_{z_{j, r}}-\boldsymbol{p}_{r}^{(j)} \neq \boldsymbol{p}_{z_{j, s}}-\boldsymbol{p}_{s}^{(j)}$ but their second coordinates coincide, i.e.

$$
\begin{aligned}
\operatorname{pr}_{2}\left(\boldsymbol{p}_{z_{j, r}}-\boldsymbol{p}_{r}^{(j)}\right) & =\operatorname{pr}_{2}\left(\boldsymbol{p}_{z_{j, s}}-\boldsymbol{p}_{s}^{(j)}\right) \\
& =\operatorname{pr}_{2}\left(I_{k}^{-n_{j, r}}\left(\boldsymbol{p}_{z_{j, r}}-\boldsymbol{p}_{r}^{(j)}\right)\right)=\operatorname{pr}_{2}\left(I_{k}^{-n_{j, s}}\left(\boldsymbol{p}_{z_{j, s}}-\boldsymbol{p}_{s}^{(j)}\right)\right) .
\end{aligned}
$$

Then, the existence of the natural number $m$ and the conclusion of the lemma itself follow by Lemma 2.15.

\section{WANDERING POINTS AS AN OBSTRUCTION TO IRRATIONAL CIRCLE FACTORS}

The main purpose of this section consists in describing the construction of three examples of totally irrational pseudo-rotations with uniformly bounded rotational deviations but which do not admit irrational circle rotations as topological factors.

In these three examples the geometry of the wandering sets plays a fundamental role, showing that the small wandering domains hypothesis is fundamental and sharp in Theorem A.

First we shall perform a general construction, which is a slight modification of classical suspensions, that will be used in $\S \S 3.2$ and 3.3 .

3.1. Suspending circle homeomorphisms. Given arbitrary homeomorphisms $g_{1}, g_{2} \in \operatorname{Homeo}_{0}(\mathbb{T})$ and a lift $\tilde{g}_{1}: \mathbb{R} \supset$ of $g_{1}$, we will construct the "time- $\tilde{g}_{1}$ of the suspension flow of $g_{2}$, " a homeomorphism $g \in$ Homeo $_{0}\left(\mathbb{T}^{2}\right)$ which is defined as follows.

First, consider the equivalence relation $\sim$ on $\mathbb{R} \times \mathbb{T}$ given by

$$
(s, x) \sim\left(s^{\prime}, x^{\prime}\right) \Longleftrightarrow s^{\prime}-s \in \mathbb{Z} \text { and } x=g_{2}^{s^{\prime}-s}\left(x^{\prime}\right) .
$$

Since $g_{2}$ is homotopic to the identity, the quotient space $(\mathbb{R} \times \mathbb{T}) / \sim$ is indeed homeomorphic to $\mathbb{T}^{2}$, and we shall just identify them without any further reference. 
As usual, we define the suspension flow $\Phi: \mathbb{R} \times \mathbb{T}^{2} \rightarrow \mathbb{T}^{2}$ by

$$
\Phi^{t}[s, x]:=[t+s, x], \quad \forall(s, x) \in \mathbb{R} \times \mathbb{T}, \forall t \in \mathbb{R},
$$

where $[s, x]$ denotes the equivalence class of the point $(s, x)$.

Then, we define the homeomorphism $g: \mathbb{T}^{2} \bigcirc$ as the "time- $\tilde{g}_{1}$ " of $\Phi$. More precisely, we write

$$
g[t, x]:=\left[\tilde{g}_{1}(t), x\right], \quad \forall(t, x) \in \mathbb{R} \times \mathbb{T},
$$

where $\tilde{g}_{1}: \mathbb{R} \oslash$ is a lift of $g_{1}$.

In order to verify that $g$ is indeed well defined, it is enough to notice that $\tilde{g}_{1}$ commutes with any integer translation on $\mathbb{R}$.

Then we have the following

Proposition 3.1. The homeomorphism $g: \mathbb{T}^{2} ๖$ we constructed above exhibits the following properties:

(i) $g$ is a skew-product, i.e. it leaves invariant the "vertical circle foliation" given by the $\{\{x\} \times \mathbb{T}: x \in \mathbb{T}\}$

(ii) $g$ is a pseudo-rotation and its rotation vector is totally irrational if and only if the numbers $1, \rho\left(\tilde{g}_{1}\right), \rho\left(\tilde{g}_{1}\right) \rho\left(\tilde{g}_{2}\right)$ are linearly independent over $\mathbb{Q}$, for any lift $\tilde{g}_{2}: \mathbb{R} \bigcirc$ of $g_{2}$;

(iii) $g$ exhibits uniformly bounded rotational deviations (i.e. condition (27) holds);

(iv) if $g_{2}$ is a Denjoy homeomorphism (i.e. it is periodic point free and $\mathscr{W}\left(g_{2}\right) \neq$ $\varnothing), \tilde{g}_{1}$ is fixed point free and $\Omega\left(g_{1}\right)=\mathbb{T}$, then it holds

$$
\mathscr{W}(g)=\bigcup_{t \in \mathbb{R}} \Phi^{t}\left(\{0\} \times \mathscr{W}\left(g_{2}\right)\right) .
$$

In particular, every connected component of the wandering set $\mathscr{W}(g)$ is liftunbounded and inessential.

(v) If $g_{1}$ and $g_{2}$ are Denjoy homeomorphisms, then

$$
\mathscr{W}(g):=\mathscr{W}\left(g_{1}\right) \times \mathbb{T} \cup \bigcup_{t \in \mathbb{R}} \Phi^{t}\left(\{0\} \times \mathscr{W}\left(g_{2}\right)\right) .
$$

In particular, $\mathscr{W}(g)$ is connected and fully essential.

(vi) when the rotation vector of $g$ is totally irrational, the homeomorphism $g$ is a topological extension of a minimal translation of $\mathbb{T}^{2}$ and $\Omega(g)$ is the only minimal set for $g$.

Proof. In order to prove (i), it is enough to notice that the vertical circle foliation $\{\{t\} \times \mathbb{T}: t \in \mathbb{R}\}$ of $\mathbb{R} \times \mathbb{T}$ is invariant by the quotient map $(t, x) \mapsto\left(t+1, g_{2}^{-1}(x)\right)$ and the flow $\widetilde{\Phi}: \mathbb{R} \times(\mathbb{R} \times \mathbb{T}) \ni(t,(s, x)) \mapsto(t+s, x)$, which is the lift of flow $\Phi$.

One can easily verify that the there exists a lift $\tilde{g}: \mathbb{R}^{2} ๖$ of $g$ such that $\rho(\tilde{g})=$ $\left\{\left(\rho\left(\tilde{g}_{1}\right), \rho\left(\tilde{g}_{1}\right) \rho\left(\tilde{g}_{2}\right)\right)\right\}$. Then this implies (ii).

To prove (iii) it is enough to notice that $g$ leaves invariant two topologically transverse foliations with different asymptotic homological directions (see [KK09, Proposition 4.1] and [KPR18, Theorem 5.4] for details): one of them is the vertical circle foliations of the skew-product structure given by (i); the other one is given by the orbits of the (singularity free) flow $\Phi$.

Properties (iv) and (v) are straightforward consequences of classical results about the non-wandering set of suspension flows.

Finally, in order to prove (vi), observe that, by (ii), if $g$ is a totally irrational pseudo rotation, then $g_{1}$ and $g_{2}$ have irrational rotation numbers. So, by classical Poincaré theory, both of them are topological extension of irrational rotations of the circle, and $\Omega\left(g_{1}\right)$ and $\Omega\left(g_{2}\right)$ are the only minimal sets for $g_{1}$ and $g_{2}$, respectively. These properties pass to the flow $\Phi$ by suspension. 
3.2. Unbounded inessential wandering set. In this paragraph we show the existence of a totally irrational pseudo-rotation $f \in \operatorname{Homeo}_{0}\left(\mathbb{T}^{2}\right)$, exhibiting uniformly bounded rotational deviations (in every direction), whose wandering set $\mathscr{W}(f)$ is connected, inessential and lift-unbounded and such that $f$ does not admit any irrational circle rotation as a topological factor.

To do that, let $g_{2}: \mathbb{T} \oslash$ be a Denjoy homeomorphism with just one orbit of wandering domains (i.e. $\operatorname{Per}\left(g_{2}\right)=\varnothing, \mathscr{W}\left(g_{2}\right) \neq \varnothing$ and $\mathscr{W}\left(g_{2}\right)=\bigcup_{n} g_{2}^{n}(I)$, for any connected component $I$ of $\left.\mathscr{W}\left(g_{2}\right)\right)$ and $\tilde{g}_{1}=T_{\rho_{1}}: \mathbb{R} \circlearrowleft$ be a translation such that the map $g \in$ Homeo $_{0}\left(\mathbb{T}^{2}\right)$ constructed in $\S 3.1$ is a totally irrational pseudo-rotation (see condition (ii) of Proposition 3.1). Since $g_{2}$ has just one orbit of wandering domains and property (iv) of Proposition 3.1 holds, we have that $\mathscr{W}(g)$ is connected.

By (vi) of Proposition 3.1, we know that $g$ is a topological extension of a minimal rotation of $\mathbb{T}^{2}$. One can easily show that two different points $z, z^{\prime} \in \mathbb{T}^{2}$ are Kronecker equivalent for $g$ (see Definition 2.2) if and only if $\operatorname{pr}_{1}(z)=\operatorname{pr}_{1}\left(z^{\prime}\right)$ and there exists a connected component $J$ of the set $\mathscr{W}(g) \cap\left\{\operatorname{pr}_{1}(z)\right\} \times \mathbb{T}$ such that $z, z^{\prime} \in \bar{J}$.

So, given any topological open disc $U \subset \mathscr{W}(g)$, we can clearly find two points $w_{0}, w_{1} \in U$ which are not Kronecker equivalent; and thus, they are in fact Kronecker separated (again see Definition 2.2 for details). Moreover, taking $U$ small enough, we can assume $U$ is a wandering set for $g$. Then, since $U$ is open and connected, there exists $\ell \in \operatorname{Homeo}_{0}\left(\mathbb{T}^{2}\right)$ so that supp $\ell \subset U \subset \mathscr{W}(g)$ and $\ell\left(w_{0}\right)=w_{1}$.

Then we just define $f:=g \circ \ell$. Since $\ell$ is supported in a $g$-wandering set, it holds $\Omega(f)=\Omega(g)$, and therefore, $f$ and $g$ coincide on this set. So, $f$ has a connected, inessential and lift-unbounded wandering set as well. Moreover, since $g$ exhibits uniformly bounded rotational deviations and $\ell$ is supported on a $g$-wandering liftbounded set, this implies $f$ exhibits uniformly bounded rotations deviations as well.

We claim that $f$ does not admit any irrational circle rotation as topological factor. To prove this, reasoning by contradiction, let us suppose there is a semiconjugacy $h: \mathbb{T}^{2} \rightarrow \mathbb{T}$ and a minimal rotation $T_{\rho}: \mathbb{T} \circlearrowleft$ such that $h \circ f=T_{\rho} \circ h$. Since $\Omega(f)$ is a minimal set for $f$, we have that two points of $\Omega(f)=\Omega(g)$ are Kronecker equivalent for $f$ if and only if so they are for $g$. Then, for each $i \in\{0,1\}$, we can consider an arbitrary point

$$
w_{i}^{\prime} \in \Omega(g) \cap \overline{\mathrm{cc}\left(\mathscr{W}(g) \cap\left\{\mathrm{pr}_{1}\left(w_{i}\right)\right\} \times \mathbb{T}, w_{i}\right)} .
$$

Since $w_{0}$ and $w_{1}$ are Kronecker separated for $g$, and $w_{i}$ and $w_{i}^{\prime}$ are $g$-proximal, for $i \in\{0,1\}$, we conclude $w_{0}^{\prime}$ and $w_{1}^{\prime}$ are Kronecker separated for $g$. So, $w_{0}^{\prime}$ and $w_{1}^{\prime}$ are Kronecker separated for $f$, too; but $w_{0}$ and $w_{1}^{\prime}$ are $f$-proximal and $w_{0}$ and $w_{0}^{\prime}$ are $f^{-1}$-proximal. This clearly contradicts the existence of an irrational circle factor.

3.3. Fully essential wandering set. In this paragraph we describe the construction of a totally irrational pseudo-rotation $f \in \operatorname{Homeo}_{0}\left(\mathbb{T}^{2}\right)$ with uniformly bounded rotational deviations, such that the wandering set $\mathscr{W}(f)$ is fully essential and such that $f$ does not admit any irrational circle factor.

The construction is very similar to that one performed in $\S 3.2$. In this case we start considering two Denjoy maps $g_{1}, g_{2} \in \operatorname{Homeo}_{0}(\mathbb{T})$, with lifts $\tilde{g}_{1}, \tilde{g}_{2}: \mathbb{R} \oslash$ such that $1, \rho\left(\tilde{g}_{1}\right)$ and $\rho\left(\tilde{g}_{1}\right) \rho\left(\tilde{g}_{2}\right)$ are linearly independent over $\mathbb{Q}$.

Then, let $g \in \operatorname{Homeo}_{0}\left(\mathbb{T}^{2}\right)$ be the homeomorphism given by the construction described at the beginning of $\S 3.1$, associated to $\tilde{g}_{1}$ and $g_{2}$. By (ii) and (v) of Proposition 3.1, we know that $g$ is a totally irrational pseudo-rotation and $\mathscr{W}(g)$ is a fully essential connected set.

Then, by $(v)$ we can choose a point $w_{0} \in \mathscr{W}(g)$ such that $\operatorname{pr}_{1}\left(w_{0}\right) \in \Omega\left(g_{1}\right)$. So we know there exist $I \subset \mathscr{W}\left(g_{2}\right), s_{0} \in \mathbb{R}$ and $y_{0} \in I$ such that

$$
w_{0}=\Phi^{s_{0}}\left(0, y_{0}\right) \in \Phi^{s_{0}}(\{0\} \times I) \text {. }
$$


Then, for any $\varepsilon>0$ sufficiently small, the open set

$$
U:=\bigcup_{\left|s-s_{0}\right|<\varepsilon} \Phi^{s}(\{0\} \times I)
$$

is a wandering set for $g$.

Since $\Omega\left(g_{1}\right)$ has no isolated points and $U$ is an open topological disc, we know there exists a real number $s_{1}$ with $0<\left|s_{1}-s_{0}\right|<\varepsilon$ such that the point $w_{1}:=$ $\Phi^{s_{1}}\left(0, y_{0}\right) \in U, \operatorname{pr}_{1}\left(w_{1}\right) \in \Omega\left(g_{1}\right)$ and there are infinitely many points in the arc flow of $\Phi$ between the points $w_{0}$ and $w_{1}$ such that their projections on the firs coordinate belongs to $\Omega\left(g_{1}\right)$, i.e. the set

$$
\left\{t \in(0,1): \operatorname{pr}_{1}\left(\Phi^{t s_{0}+(1-t) s_{1}}\left(0, y_{0}\right)\right) \in \Omega\left(g_{1}\right)\right\}
$$

is not empty, (and in fact, $s_{1}$ can be chosen such that this set is infinite).

Now observe that given any point $y \in \Omega\left(g_{2}\right) \subset \mathbb{T}$ and any pair of real numbers $s<s^{\prime}$, we have that $\Phi^{s}(0, y)$ and $\Phi^{s^{\prime}}(0, y)$ are Kronecker separated if an only if there exists $t \in\left(s, s^{\prime}\right)$ such that $\operatorname{pr}_{1}\left(\Phi^{t}(0, y)\right) \in \Omega\left(g_{1}\right)$.

So, if $y^{\prime} \in \Omega\left(g_{2}\right)$ be any end point of the interval $I \subset \mathscr{W}\left(g_{2}\right)$ we considered above, we know that the points $\Phi^{s_{i}}\left(0, y^{\prime}\right)$ and $w_{i}=\Phi^{s_{i}}\left(0, y_{0}\right)$ are $g$-proximal, for $i=0,1$. Due to our previous remark and property $(43)$, we know that the points $\Phi^{s_{0}}\left(0, y^{\prime}\right)$ and $\Phi^{s_{1}}\left(0, y^{\prime}\right)$ are Kronecker separated, and consequently, the points $w_{0}$ and $w_{1}$ are Kronecker separated as well.

As we did in $\S 3.2$, we consider a homeomorphism $\ell \in \operatorname{Homeo}_{0}\left(\mathbb{T}^{2}\right)$ such that $\ell\left(w_{0}\right)=w_{1}$ and supp $\ell \subset U \subset \mathscr{W}(g)$. Again, we define $f:=g \circ \ell$. By the very same argument we exposed in $\S 3.2$, one can show $f$ is a totally irrational pseudo-rotation exhibiting uniformly bounded rotational deviations, fully essential wandering set and having no irrational circle factor.

3.4. Inessential bounded non-small wandering domains. In this paragraph we describe the construction of a totally irrational pseudo-rotation $f: \mathbb{T}^{2} ๖$ not admitting any irrational circle factor, and such that it exhibits uniformly bounded rotational deviations, its wandering set is the union of countably many inessential lift-bounded wandering domains, all of them having the same diameter and hence, not satisfying the small wandering domain hypothesis (see Definition 2.3).

To do that, let us start by considering a totally irrational vector $\alpha \in \mathbb{R}^{2}$ and let $T_{\alpha}: \mathbb{T}^{2} \bigcirc$ be the corresponding rigid rotation. Given any $\gamma \in \mathbb{R} \backslash \mathbb{Q}$ and $\delta>0$, let us define

$$
\mathscr{F}_{\delta}^{\gamma}:=\pi\left\{(t, t \gamma) \in \mathbb{R}^{2}: t \in(-\delta, \delta)\right\} \subset \mathbb{T}^{2} .
$$

Notice that fixing the totally irrational vector $\alpha \in \mathbb{R}^{2}$, there exists $\delta_{0}>0$ such that

$$
T_{\alpha}^{n}\left(\mathscr{F}_{\delta}^{\gamma}\right) \cap \mathscr{F}_{\delta}^{\gamma}=\varnothing, \quad \forall \delta \in\left(0, \delta_{0}\right), \quad \forall n \in \mathbb{Z} \backslash\{0\}
$$

Then let us fix such a $\delta$.

By classical "à la Denjoy" surgery procedures, we can construct a topological extension $g \in \mathrm{Homeo}_{0}\left(\mathbb{T}^{2}\right)$ of $T_{\alpha}$ satisfying the following properties: there exist a continuous map $h: \mathbb{T}^{2} \oslash$ in the identity homotopy class and a sequence $\left\{\alpha_{n}\right\}_{n \in \mathbb{Z}}$ of points of $\mathbb{T}^{2}$ such that $h \circ g=T_{\alpha} \circ h$, each fiber $h^{-1}(z)$ is a singleton if and only if $z \in \mathbb{T}^{2} \backslash \bigcup_{n \in \mathbb{Z}} T_{\alpha}^{n}\left(\mathscr{F}_{\delta}^{\gamma}\right)$, and

$$
h^{-1}(z)=\left\{z+\alpha_{n}+\pi(-\gamma t, t): t \in\left(-\delta_{n}(z), \delta_{n}(z)\right)\right\},
$$

whenever $z \in T_{\alpha}^{n}\left(\mathscr{F}_{\delta}^{\gamma}\right)$, and where $\delta_{n}(z):=2^{-|n|-10}\left(\delta-d_{\mathbb{T}^{2}}\left(z, T_{\alpha}^{n}(0)\right)\right)$ and $d_{\mathbb{T}^{2}}(\cdot, \cdot)$ denotes the distance function given by (10). 
Notice that the wandering set of $g$ is given by

$$
\mathscr{W}(g)=\bigsqcup_{n \in \mathbb{Z}} h^{-1}\left(T_{\alpha}^{n}\left(\mathscr{F}_{\delta}^{\gamma}\right)\right),
$$

where $\bigsqcup$ denotes the disjoint union operator, and

$$
\operatorname{diam}\left(h^{-1}\left(T_{\alpha}^{n}\left(\mathscr{F}_{\delta}^{\gamma}\right)\right)\right)=2 \delta, \quad \forall n \in \mathbb{Z} .
$$

So, $g$ does not satisfies the small wandering domain hypothesis given by Definition 2.3.

Then observe that two different points of $w_{0}, w_{1} \in \mathbb{T}^{2}$ are Kronecker equivalent for $g$ if and only if there exist $n \in \mathbb{Z}, z \in T_{\alpha}^{n}\left(\mathscr{F}_{\delta}^{\gamma}\right)$ and $t_{0}, t_{1} \in\left[-\delta_{n}(z), \delta_{n}(z)\right]$ satisfying

$$
w_{i}=z+\alpha_{n}+\pi\left(-\gamma t_{i}, t_{i}\right), \quad \text { for } i \in\{0,1\} .
$$

In particular, inside the wandering domain $h^{-1}\left(\mathscr{F}_{\delta}^{\gamma}\right)$ we can find two points $w_{0}$ and $w_{1}$ which are not Kronecker equivalent. Moreover, since $\gamma$ is irrational, this implies $w_{0}$ and $w_{1}$ are indeed Kronecker separated.

Then, as we did in $\S \S 3.2$ and 3.3, and observing $h^{-1}\left(\mathscr{F}_{\delta}^{\gamma}\right)$ is an open topological disc, we can find a homeomorphism $\ell \in \operatorname{Homeo}_{0}\left(\mathbb{T}^{2}\right)$ such that $h\left(w_{0}\right)=w_{1}$ and $\operatorname{supp} \ell \subset h^{-1}\left(\mathscr{F}_{\delta}^{\gamma}\right)$. Then we define $f:=g \circ \ell$.

Again, $g$ clearly exhibits uniformly bounded rotational deviations and $\ell$ is supported on a lift-bounded wandering domain, so $f$ exhibits uniformly bounded rotational deviations, too. Regarding the wandering set, it clearly holds $\mathscr{W}(f)=\mathscr{W}(g)$. So, $f$ does not satisfy the small wandering condition either. There exist points $w_{i}^{\prime} \in \Omega(g)$ such that $w_{i}$ is Kronecker equivalent to $w_{i}^{\prime}$, for $i \in\{0,1\}$ and $w_{0}$ is $f$ proximal to $w_{1}^{\prime}$ and $f^{-1}$-proximal to $w_{0}^{\prime}$, which are Kronecker separated points. So, $f$ does not admit any irrational circle factor.

\section{Proofs of Corollaries 1.1 And 1.2}

In this section we prove both corollaries assuming Theorem A.

Proof of Corollary 1.1. Since $f$ is a totally irrational pseudo-rotation, we know it is non-eventually annular. On the other hand, since $f$ exhibits uniformly bounded rotational deviations, i.e. condition (27) holds, we know that $f$ satisfies the hypotheses of Theorem A, for every $v \in \mathbb{S}^{1}$ of rational slope.

Then, we apply Theorem A twice in order to construct a "horizontal" and a "vertical" irrational circle factor, i.e. we first consider $v=(1,0)$ and then $v=(0,1)$ to get two continuous semi-conjugacies $h_{i}: \mathbb{T}^{2} \rightarrow \mathbb{T}$, with $i=1,2$, such that

$$
h_{i} \circ f=T_{\rho_{i}} \circ h_{i}, \quad \text { for } i=1,2,
$$

where $\rho(\tilde{f})=\left(\rho_{1}, \rho_{2}\right) \in \mathbb{R}^{2}$ is the rotation vector of $\tilde{f}$, and $T_{\rho_{i}}: \mathbb{T} \oslash$ is the corresponding irrational circle rotation.

Finally we just define $H: \mathbb{T}^{2} ๖$ by

$$
H(x, y):=\left(h_{1}(x), h_{2}(y)\right), \quad \forall(x, y) \in \mathbb{T}^{2},
$$

and one can easily check that $H \circ f=T_{\rho(\tilde{f})} \circ H$, as desired.

Proof of Corollary 1.2. If $f$ is a topological extension of an irrational circle rotation, then by Lemma 2.10 we know that given any lift $\tilde{f}: \mathbb{R}^{2} \bigcirc$ of $f$, there exit $C>0$, $v \in \mathbb{S}^{1}$ and $\rho \in \mathbb{R} \backslash \mathbb{Q}$ such that the estimate of that lemma holds. Since $f$ is homotopic to a Dehn twist $I_{k}$ with $k \neq 0$, we conclude that $v$ is equal to $\pm(0,1)$, and so, estimate (3) holds. 
Reciprocally, if (3) holds and taking into account $f$ is homotopic to a Dehn twist, we conclude $f$ is not eventually annular and hence we can directly apply Theorem A to conclude the existence of an irrational circle factor.

\section{5. $\rho$-CENTRALIZED SKEW-PRODUCT}

In this section we introduce the main character of this work: the $\rho$-centralized skew-product induced by the torus homeomorphism $f$. This is a generalization of that one we introduced in [Koc16, KPR18] to study rotational deviations for periodic point free homeomorphisms in the identity homotopy class.

The intuitive idea behind the definition of this skew-product is to separate the rotational component of a given torus homeomorphism from its complement which is responsible for the sub-linear rotational deviations. This idea can be easily formalized regarding the group of area-preserving 2-torus homeomorphisms which are homotopic to the identity. In fact, this group can be written as the semi-direct product of the group of rotations (which is isomorphic to $\mathbb{T}^{2}$ itself) and the group of Hamiltonian homeomorphisms, i.e. the group of area-preserving homeomorphisms in the identity homotopy class with vanishing Lebesgue rotation vector (also called flux in symplectic geometry); and this group-theoretically decomposition can be taken to the dynamical system setting defining a skew-product homeomorphism on $\mathbb{T}^{2} \times \mathbb{R}^{2}$ which is just a rotation on the base and acts by (lifts of) Hamiltonian homeomorphisms on the fibers.

The main heuristic of this skew-product can be summarized as follows: bounded orbits of this skew-product correspond to orbits of the original homeomorphism with bounded rotational deviations with respect to the rotation vector that acts on the base of the skew-product. The reader can find in [Koc16, §6.1] all the details of this construction, especially those relating the semi-direct group decomposition of the group of area-preserving 2-torus homeomorphism and the definition of the induced skew-product, that we called fiberwise Hamiltonian skew-product there. Later, in [KPR18] we extended this construction for any homeomorphism in the identity isotopy class and any $\rho \in \mathbb{R}^{2}$, and we started calling them $\rho$-centralized skew-product, which is the name we shall continue using from now on.

In this work we will introduce a slight modification of the construction we performed in [KPR18] in order to deal with homeomorphisms in Dehn twist isotopy classes. In fact, as we saw in $\$ 2.4 .1$, when the homeomorphism $f \in \operatorname{Homeo}_{k}\left(\mathbb{T}^{2}\right)$ with $k \neq 0$, one cannot define rotation vectors as elements of $\mathbb{R}^{2}$, but just vertical rotation numbers as given by (16). So, here $\rho$-centralized skew-products will be defined on $\mathbb{T}^{2} \times \mathbb{A}$ and not on $\mathbb{T}^{2} \times \mathbb{R}^{2}$ as done in [Koc16, KPR18].

Summarizing the results we are going to find in this section, let $f \in \operatorname{Homeo}_{k}\left(\mathbb{T}^{2}\right)$ be a given homeomorphism and $F: \mathbb{T}^{2} \times \mathbb{A} \bigcirc$ denote the induced $\rho$-centralized skew-product (that we will formally introduce just after this brief summary). In Proposition 5.1 we study the group of symmetries of $F$, showing it includes a full flow called $\Gamma$. Then, in Proposition 5.4 we prove that there is a close relation between points of $\mathbb{T}^{2}$ exhibiting bounded vertical rotational deviations for $f$ and points of $\mathbb{T}^{2} \times \mathbb{A}$ whose $F$-orbits are bounded. One of the main results of this section is Theorem 5.5 where we show that under the $\Omega$-recurrence hypothesis, there is natural correspondence between non-wandering points $f$ and non-wandering points of $F$, showing in particular that $f$ is a non-wandering homeomorphism if and only if $F$ is. Finally, in Theorem 5.6 we study the topology of $F$-invariant bounded connected sets, showing that under the hypotheses of Theorem A, the complement of theses sets have exactly two unbounded connected components. The boundary of these sets will be used in $\S 6$ to construct the fibers of the semi-conjugacy with the irrational circle factor. 
Let us state now the precise definition of the induced $\rho$-centralized skew-product. Let $k$ be any integer number, $f \in \operatorname{Homeo}_{k}\left(\mathbb{T}^{2}\right)$ an arbitrary homeomorphism and $\tilde{f}: \mathbb{R}^{2} \bigcirc$ be a lift of $f$. We know that the displacement function $\Delta_{\tilde{f}}:=\tilde{f}-I_{k}: \mathbb{R}^{2} ๖$ is $\mathbb{Z}^{2}$-periodic, and thus, it can be considered as an element of $C^{0}\left(\mathbb{T}^{2}, \mathbb{R}^{2}\right)$, where $I_{k} \in \operatorname{SL}(2, \mathbb{Z})$ is given by $(12)$.

For the sake of simplicity, let us write

$$
\Delta_{i}:=\operatorname{pr}_{i} \circ \Delta_{\tilde{f}}, \quad \text { for } i=1,2 .
$$

On the other hand, let $\hat{f}: \mathbb{A} \bigcirc$ be the only annulus homeomorphism such that $\tilde{\pi} \circ \tilde{f}=\hat{f} \circ \tilde{\pi}$, where $\tilde{\pi}$ is the covering map given by (17).

Then, given any $\rho \in \mathbb{R}$ let us define the $\rho$-centralized skew-product induced by $\tilde{f}$ as the homeomorphism $F: \mathbb{T} \times \mathbb{A} \bigcirc$ given by

$$
F(t, x, \tilde{y}):=\left(T_{\rho}(t), x+k(y+t)+\pi\left(\Delta_{1}(x, y+t)\right), \tilde{y}+\Delta_{2}(x, y+t)-\rho\right),
$$

for every $(t, x, \tilde{y}) \in \mathbb{T} \times \mathbb{A}=\mathbb{T} \times \mathbb{T} \times \mathbb{R}$ and where $y:=\pi(\tilde{y}) \in \mathbb{T}$.

The usefulness of map $F$ can be briefly summarized with the following simple but important property:

$$
F^{n}(t, \hat{z})=\left(T_{\rho}^{n}(t), \hat{T}_{\tilde{t}}^{-1} \circ\left(\hat{T}_{\rho}^{-n} \circ \hat{f}^{n}\right) \circ \hat{T}_{\tilde{t}}(\hat{z})\right),
$$

for any $(t, \hat{z}) \in \mathbb{T} \times \mathbb{A}$, any $\tilde{t} \in \pi^{-1}(t)$ and every $n \in \mathbb{Z}$.

On the other hand, let us notice that the skew-product $F$ has a full flow of symmetries: for each $s \in \mathbb{R}$ consider the map $\Gamma^{s}: \mathbb{T} \times \mathbb{A} \oslash$ given by $\Gamma^{s}:=T_{s} \times \hat{T}_{-s}$, i.e.

$$
\Gamma^{\mathcal{S}}(t, x, \tilde{y}):=(t+\pi(s), x, \tilde{y}-s)=\left(T_{\mathcal{S}}(t), \hat{T}_{-s}(x, \tilde{y})\right), \quad \forall(t, x, \tilde{y}) \in \mathbb{T} \times \mathbb{A} .
$$

Then, we have the following

Proposition 5.1. The map $\Gamma: \mathbb{R} \times \mathbb{T} \times \mathbb{A} \rightarrow \mathbb{T} \times \mathbb{A}$ is a flow and, for each $s \in \mathbb{R}$, the homeomorphisms $\Gamma^{\mathcal{S}}$ and $F$ commute, and $\Gamma^{\mathcal{S}}$ is an isometry of the metric space $\left(\mathbb{T} \times \mathbb{A}, d_{\mathbb{T} \times \mathbb{A}}\right)$, where the distance function is given by

$$
d_{\mathbb{T} \times \mathbb{A}}\left((t, z),\left(t^{\prime}, z^{\prime}\right)\right)=d_{\mathbb{T}}\left(t, t^{\prime}\right)+d_{\mathbb{A}}\left(z, z^{\prime}\right), \quad \forall(t, z),\left(t^{\prime}, z^{\prime}\right) \in \mathbb{T} \times \mathbb{A} .
$$

Proof. This follows from straightforward computations. In fact,

$$
\Gamma^{s+s^{\prime}}=T_{s+s^{\prime}} \times \hat{T}_{-s-s^{\prime}}=\left(T_{s} \times \hat{T}_{-s}\right) \circ\left(T_{s^{\prime}} \times \hat{T}_{-s^{\prime}}\right)=\Gamma^{s} \circ \Gamma^{s^{\prime}}, \quad \forall s, s^{\prime} \in \mathbb{R} .
$$

So, given any $s \in \mathbb{R}$ and any $(t, x, \tilde{y}) \in \mathbb{T} \times \mathbb{A}$, it holds

$$
\begin{aligned}
& F\left(\Gamma^{S}(t, x, \tilde{y})\right)=F(t+\pi(s), x, \tilde{y}-s) \\
& =\left(T_{\rho}\left(T_{S}(t)\right), x+k(y+t)+\pi \circ \Delta_{1}(x, y+t), \tilde{y}-s+\Delta_{2}(x, y+t)-\rho\right) \\
& =\Gamma^{S}(F(t, x, \tilde{y})) .
\end{aligned}
$$

Finally, $\Gamma^{S}$ is an isometry because $T_{S}$ is an isometry of $\left(\mathbb{T}, d_{\mathbb{T}}\right)$ and $\hat{T}_{\mathcal{S}}$ of $\left(\mathbb{A}, d_{\mathbb{A}}\right)$.

Then, let us fix some terminology. Given any $(t, \hat{z}) \in \mathbb{T} \times \mathbb{A}$, the $\Gamma$-line through $(t, \hat{z})$ is its flow line, i.e. it is given by

$$
\Gamma(t, \hat{z}):=\left\{\Gamma^{\mathcal{S}}(t, \hat{z}) \in \mathbb{T} \times \mathbb{A}: s \in \mathbb{R}\right\} .
$$

On the other hand, we introduce the concept of blocks of $\mathbb{T} \times \mathbb{A}$, which are a particular kind of open subsets of $\mathbb{T} \times \mathbb{A}$ : given an open set $V \subset \mathbb{A}$, a point $t \in \mathbb{T}$ 
and a positive real number $r$, we define the corresponding $r$-block centered at $t$ associated to $V$ by

$$
V^{r, t}:=\bigcup_{|s|<r} \Gamma^{\mathcal{S}}(\{t\} \times V) \subset \mathbb{T} \times \mathbb{A} .
$$

A rather simple but important property of blocks is given by the following

Proposition 5.2. If $V \subset \mathbb{A}$ is an open subset, $t \in \mathbb{T}$ and $r>0$, and $V^{r, t}$ is the $r$-block centered at $t$ associated to $V$ given by (47), then it holds

$$
F^{n}\left(V^{r, t}\right)=\left(\hat{T}_{\tilde{t}}^{-1} \circ \hat{T}_{\rho}^{-n} \circ \hat{f}^{n} \circ \hat{T}_{\tilde{t}}(V)\right)^{r, T_{\rho}^{n}(t)}, \quad \forall n \in \mathbb{Z}, \forall \tilde{t} \in \pi^{-1}(t) .
$$

In particular, the F-image of any r-block is another $r$-block.

Proof. It easily follows from (45), Proposition 5.1 and (47).

Finally, as a straightforward consequence of (45) we get the following

Proposition 5.3. Given any $z \in \mathbb{T}^{2}$, any $n \in \mathbb{Z}$, any $\hat{z} \in \hat{\pi}^{-1}(z)$ and any $\hat{w} \in$ $\hat{\pi}^{-1}\left(f^{n}(z)\right)$ (where projection $\hat{\pi}$ is given by $\left.(18)\right)$, the point $F^{n}(0, \hat{z})$ belongs to the $\Gamma$-line through $(0, \hat{w})$.

Proof. Since $\hat{\pi} \circ \hat{f}=f \circ \hat{\pi}$, we have

$$
\hat{\pi}\left(\hat{f}^{n}(\hat{z})\right)=f^{n}(\hat{\pi}(\hat{z}))=f^{n}(z)=\hat{\pi}(\hat{w}) .
$$

So there exists $m \in \mathbb{Z}$ such that $\hat{T}_{m}(\hat{w})=\hat{f}^{n}(\hat{z})$, or equivalently we can write

$$
\Gamma^{-m}(0, \hat{w})=\left(0, \hat{f}^{n}(\hat{z})\right) .
$$

Then, by (45) we know that

$$
F^{n}(0, \hat{z})=\left(n \pi(\rho), \hat{T}_{\rho}^{-n} \circ \hat{f}^{n}(\hat{z})\right)=\Gamma^{n \rho}\left(0, \hat{f}^{n}(\hat{z})\right)=\Gamma^{n \rho-m}(0, \hat{w}) .
$$

5.1. The $\rho$-centralized skew-product and rotational deviations. From now on and until the end of this section we suppose $\tilde{f}: \mathbb{R}^{2} \bigcirc$ is a lift of a homeomorphism $f \in \operatorname{Homeo}_{k}\left(\mathbb{T}^{2}\right)$ exhibiting uniformly bounded vertical deviations, with $\rho, C$ and $v=(0,1)$ as in $(23)$. Let $F: \mathbb{T} \times \mathbb{A} \bigcirc$ be the $\rho$-centralized skew-product induced by $\tilde{f}$ as defined in (44).

Then we have the following

Proposition 5.4. Every F-orbit is bounded in the vertical direction, i.e. if $(t, x, \tilde{y}) \in$ $\mathbb{T} \times A$ is an arbitrary point and we define $\left(t_{n}, x_{n}, \tilde{y}_{n}\right):=F^{n}(t, x, \tilde{y})$, then it holds

$$
\left|\tilde{y}_{m}-\tilde{y}_{n}\right| \leqslant 2 C, \quad \forall m, n \in \mathbb{Z},
$$

where $C$ is the constant given by (23).

Proof. This is a straightforward consequence of estimate (23) and property (45).

The following result will play a key role in our work:

Theorem 5.5. If $f$ is $\Omega$-recurrent (see Definition 2.1), then a point $(x, y) \in \mathbb{T}^{2}$ is non-wandering for $f$ if and only if $\Gamma^{\mathcal{S}}(0, x, \tilde{y}) \in \Omega(F)$, for every $\tilde{y} \in \pi^{-1}(y)$ and all $s \in \mathbb{R}$.

Proof. First observe that, by Proposition 5.1, the set $\Omega(F)$ is $\Gamma$-invariant. So it is enough to show that a point $(x, y) \in \Omega(f)$ if and only if $(0, x, \tilde{y}) \in \Omega(F)$, for some $\tilde{y} \in \pi^{-1}(y)$.

Then, let us prove the "if" direction, which holds without the boundedness of rotational deviations and the $\Omega$-recurrence assumptions. So, let $(0, x, \tilde{y})$ be any point of $\Omega(F)$. 
Let us fix a positive number $\delta$ and write just $B$ for the open ball $B_{\delta}(x, \tilde{y}) \subset \mathbb{A}$. Then, since $(0, x, \tilde{y})$ is a non-wandering point, there exists $n \geqslant 1$ such that

$$
F^{n}\left(B^{\delta, 0}\right) \cap B^{\delta, 0} \neq \varnothing,
$$

where $B^{\delta, 0}$ denotes the $\delta$-block centered at $0 \in \mathbb{T}$ and associated to $B$, as defined by (47). So, there are $s, s^{\prime} \in(-\delta, \delta)$ such that $T_{\rho}^{n}(\pi(s))=\pi\left(s^{\prime}\right)$ and the sets $F^{n}\left(B^{\delta, 0}\right)$ and $B^{\delta, 0}$ intersect on the fiber $\left\{s^{\prime}\right\} \times \mathbb{A}$. Thus, by (45), we have

$$
\hat{T}_{s}^{-1} \circ \hat{T}_{\rho}^{-n} \circ \hat{f}^{n} \circ \hat{T}_{s}\left(\hat{T}_{-s}(B)\right) \cap \hat{T}_{-s^{\prime}}(B) \neq \varnothing,
$$

and hence,

$$
\hat{f}^{n}(B) \cap \hat{T}_{n \rho+s-s^{\prime}}(B) \neq \varnothing .
$$

However, since $T_{\rho}^{n}(\pi(s))=\pi\left(s^{\prime}\right)$, we know there exists $p \in \mathbb{Z}$ such that $n \rho+s=$ $s^{\prime}+p$, and then, recalling $B$ denotes the ball of radius $\delta$ and center $(x, \tilde{y})$ in $\mathbb{A}$, we get

$$
f^{n}\left(B_{\delta}(x, y)\right) \cap B_{\delta}(x, y) \neq \varnothing,
$$

where $y=\pi(\tilde{y})$. Therefore, $(x, y) \in \Omega(f)$, as desired.

Now, let $(x, y)$ be any point of $\Omega(f)$ and let us fix a point $\tilde{y} \in \pi^{-1}(y)$. Given any real number $r>0$, let $B_{r}$ denote the open ball $B_{r}(x, \tilde{y}) \subset \mathbb{A}$ and $B_{r}^{r, 0}$ be the $r$-block centered at 0 associated to $B_{r}$. Observe the family of blocks $\left\{B_{r}^{r, 0}: r>0\right\}$ is a local base of neighborhoods at the point $(0, x, \tilde{y})$. So, let us fix a real number $r>0$ that, without loss of generality, we can suppose is less than $1 / 4$, and let us show there exists $n \in \mathbb{N}$ such that $B_{r}^{r, 0} \cap F^{-n}\left(B_{r}^{r, 0}\right) \neq \varnothing$.

Since $f$ is $\Omega$-recurrent, by Lemma 2.1 there exists an $f$-recurrent point $\left(x^{\prime}, y^{\prime}\right) \in$ $B_{r}(x, y)$ and a strictly increasing sequence of positive integers $\left(n_{j}\right)_{j \geqslant 1}$ such that

$$
\left(x_{j}^{\prime}, y_{j}^{\prime}\right):=f^{n_{j}}\left(x^{\prime}, y^{\prime}\right) \in B_{r}(x, y), \quad \forall j \in \mathbb{N} .
$$

Since $r<1 / 4$, there is a unique point $\tilde{y}^{\prime} \in \pi^{-1}\left(y^{\prime}\right) \cap B_{r}(\tilde{y})$, and for each $j \geqslant 1$, a unique point $\tilde{y}_{j}^{\prime} \in \pi^{-1}\left(y_{j}^{\prime}\right) \cap B_{r}(\tilde{y})$.

By Proposition 5.3, for each $j \geqslant 1$ there is a real number $s_{j}$ such that

$$
F^{n_{j}}\left(0, x^{\prime}, \tilde{y}^{\prime}\right)=\Gamma^{s_{j}}\left(0, x_{j}^{\prime}, \tilde{y}_{j}^{\prime}\right), \quad \forall j \geqslant 1 .
$$

On the other hand, by Proposition 5.4 we know that

$$
\left|s_{j}\right| \leqslant 2 C, \quad \forall j \in \mathbb{N} \text {. }
$$

Now, invoking Proposition 5.1 we get

$$
F^{-n_{j}}\left(\bigcup_{|s|<r} \Gamma^{S}\left(0, x_{j}^{\prime}, \tilde{y}_{j}^{\prime}\right)\right)=\bigcup_{|s|<r} \Gamma^{S}\left(F^{-n_{j}}\left(0, x_{j}^{\prime}, \tilde{y}_{j}^{\prime}\right)\right) \subset \bigcup_{|s| \leqslant 2 C+r} \Gamma^{S}\left(0, x^{\prime}, \tilde{y}^{\prime}\right),
$$

for every $j \geqslant 1$.

Now, recalling $\Gamma$ is an isometric flow (i.e. for every $s, \Gamma^{s}$ leaves invariant the distance $d_{\mathbb{T} \times \mathbb{A}}$ defined in Proposition 5.1), we observe the arc on the right side of the equation has finite length. On the other hand, on the left side of the equation, we have infinitely many constant length segments. So we can conclude there exist $k>j \geqslant 1$ such that

$$
F^{-n_{k}}\left(\bigcup_{|s|<r} \Gamma^{\mathcal{S}}\left(0, x_{k}^{\prime}, \tilde{y}_{k}^{\prime}\right)\right) \cap F^{-n_{j}}\left(\bigcup_{|s|<r} \Gamma^{\mathcal{S}}\left(0, x_{j}^{\prime}, \tilde{y}_{j}^{\prime}\right)\right) \neq \varnothing .
$$

Since $\left(0, x_{j}^{\prime}, \tilde{y}_{j}^{\prime}\right),\left(0, x_{k}^{\prime}, \tilde{y}_{k}^{\prime}\right) \in B_{r}^{r, 0}$, this implies $F^{n_{k}-n_{j}}\left(B_{r}^{r, 0}\right) \cap B_{r}^{r, 0} \neq \varnothing$, with $n_{k}-n_{j}>$ 0 . So, $(0, x, \tilde{y}) \in \Omega(F)$.

Now we can state the main result of this section: 
Theorem 5.6. Let us suppose $f \in \operatorname{Homeo}_{k}\left(\mathbb{T}^{2}\right)$ is $\Omega$-recurrent, periodic point free, exhibits uniformly bounded vertical rotational deviations and is not eventually annular. Let $V \subset \mathbb{T} \times \mathbb{A}$ be a nonempty connected bounded open set such that $V \cap \Omega(F) \neq \varnothing$. Then, for each $t \in \mathbb{T}$, the open set

$$
\mathbb{A} \backslash{\overline{\mathscr{U}_{F}(V)}}_{t}
$$

has exactly two unbounded connected components, where $\mathscr{U}_{f}(V) \subset \mathbb{T} \times \mathbb{A}$ is the set given by (9) and $\mathscr{U}_{F}(V)_{t}$ denotes the fiber of $\mathscr{U}_{F}(V)$ over $t$ given by (6).

As a consequence of this, one concludes the set $\mathscr{U}_{F}(V)$ is in fact F-invariant.

In order to prove Theorem 5.6, let us start considering the following

Lemma 5.7. If $f \in \operatorname{Homeo}_{0}\left(\mathbb{T}^{2}\right)$ is an $\Omega$-recurrent totally irrational pseudorotation exhibiting uniformly bounded rotational deviations, i.e. estimate (27) holds, and $V$ is as in Theorem 5.6, then $\mathscr{U}_{F}(V)_{t} \subset \mathbb{A}$ is an annular set, for every $t \in \mathbb{T}$.

Proof of Lemma 5.7. Let $\tilde{f}: \mathbb{R}^{2} ๖$ be a lift of $f$ and $\bar{\rho} \in \mathbb{R}^{2}$ be the totally irrational vector appearing in estimate $(27)$. So, $\rho=\operatorname{pr}_{2}(\bar{\rho})$ is the irrational number we are considering to define the $\rho$-centralized skew-product given by (44).

Reasoning by contradiction, suppose there exists a nonempty open connected subset $V \subset \mathbb{T} \times \mathbb{A}$ and $t \in \mathbb{T}$ such $\mathscr{U}_{F}(V)_{t}$ is not annular, i.e. is inessential in $\mathbb{A}$. Since $\rho$ is irrational and $\mathscr{U}_{F}(V)$ is open, we conclude $\mathscr{U}_{F}(V)_{t}$ is inessential in $\mathbb{A}$, for every $t \in \mathbb{T}$.

Consider the covering map $\pi \times \tilde{\pi}: \mathbb{R} \times \mathbb{R}^{2} \rightarrow \mathbb{T} \times \mathbb{A}$ given by

$$
\pi \times \tilde{\pi}(\tilde{t}, \tilde{z}):=(\pi(\tilde{t}), \tilde{\pi}(\tilde{z})), \quad \forall(\tilde{t}, \tilde{z}) \in \mathbb{R} \times \mathbb{R}^{2},
$$

and let $\widetilde{\mathscr{U}_{f}(V)} \subset \mathbb{R}^{3}$ be a connected component of $(\pi \times \tilde{\pi})^{-1}\left(\mathscr{U}_{F}(V)\right)$. Again by irrationality of $\rho$, it must hold $\operatorname{pr}_{1}\left(\mathscr{U}_{F}(V)\right)=\mathbb{T}$. On the other hand, since $\pi \times \tilde{\pi}$ is a covering map, and both sets $\mathscr{U}_{F}(V)$ and $\widetilde{\mathscr{U}_{F}(V)}$ are open and connected, we get

$$
\pi \times \tilde{\pi}\left(\widetilde{\mathscr{U}_{F}(V)}\right)=\mathscr{U}_{F}(V) \text {. }
$$

So we have $\operatorname{pr}_{1}\left(\widetilde{\mathscr{U}_{F}(V)}\right)=\mathbb{R}$.

On the other hand, since $\mathscr{U}_{F}(V)$ is open, connected and bounded in $\mathbb{T} \times \mathbb{A}$, we know that $\operatorname{pr}_{3}\left(\widetilde{\mathscr{U}_{F}(V)}\right)$ is also bounded in $\mathbb{R}$. Hence, there exists $\ell \in \mathbb{Z}$, which is unique and independent of the choice of the connected component $\widetilde{\mathscr{U}_{F}(V)}$, such that

$$
\left.{\widetilde{\mathscr{U}_{F}(V)}}_{\tilde{t}+1}=T_{(\ell, 0)}\left(\widetilde{\mathscr{U}_{F}(V)}\right)_{\tilde{t}}\right), \quad \forall \tilde{t} \in \mathbb{R} .
$$

Since $V$ intersects $\Omega(F)$ and $(50)$ holds, there is $(\tilde{t}, \tilde{z}) \in \widetilde{\mathscr{U}_{F}(V)}$ so that the point $(t, z):=\pi \times \tilde{\pi}(\tilde{t}, \tilde{z})$ belongs to $\Omega(F)$. This implies there exists a sequence of positive integers $\left(n_{j}\right)_{j \geqslant 1}$ and a sequence of points $\left(\left(t_{j}, z_{j}\right)\right)_{j \geqslant 1}$ such that $\left(t_{j}, z_{j}\right) \in B_{1 / 4 j}(t, z) \subset$ $\mathbb{T} \times \mathbb{A}$ and $F^{n_{j}}\left(t_{j}, z_{j}\right) \in B_{1 / 4 j}(t, z)$, for each $j \geqslant 1$. Observe, since $F$ is periodic point free, it necessarily holds $n_{j} \rightarrow+\infty$, as $j \rightarrow \infty$. So, by (45), we can conclude that for each $j \geqslant 1$ there exist unique numbers $m_{j}, p_{j} \in \mathbb{Z}$ such that $\left|m_{j}-n_{j} \rho\right|<1 / 2 j$ and

$$
\frac{1}{n_{j}}\left\|\tilde{f}^{n_{j}}\left(\tilde{z}_{j}+\left(0, \tilde{t}_{j}\right)\right)-\tilde{z}_{j}-\left(0, \tilde{t}_{j}\right)-n_{j}(0, \rho)-\left(p_{j}, 0\right)\right\| \leqslant \frac{1}{2 j n_{j}},
$$

where $\left(\tilde{t}_{j}, \tilde{z}_{j}\right)$ is the only point in $(\pi \times \tilde{\pi})^{-1}\left(t_{j}, z_{j}\right) \cap B_{1 / j}(\tilde{t}, \tilde{z})$, provided $j \geqslant 4$.

Now, recalling we are assuming $f$ is a pseudo-rotation and $\rho(\tilde{f})=\{\bar{\rho}\}$ is the rotation set given by (14), as a consequence of (52) we get $p_{j} / n_{j} \rightarrow \operatorname{pr}_{1}(\bar{\rho})=\rho$ and $m_{j} / n_{j} \rightarrow \operatorname{pr}_{2}(\bar{\rho})$, as $j \rightarrow \infty$. 
However observe that, by (51), $p_{j}=\ell m_{j}$, for all $j \geqslant 1$. This implies that $\operatorname{pr}_{1}(\bar{\rho})=\ell \operatorname{pr}_{2}(\bar{\rho})$, contradicting the fact that $\bar{\rho}$ was totally irrational. So, $\mathscr{U}_{F}(V)_{t}$ is annular for every $t \in \mathbb{T}$.

Then we can finish the proof of Theorem 5.6:

Proof of Theorem 5.6. If $f \in \operatorname{Homeo}_{0}\left(\mathbb{T}^{2}\right)$ is a pseudo-rotation and exhibits uniformly bounded horizontal deviations, recalling vertical deviations are uniformly bounded as well, then it exhibits uniformly bounded rotational deviations in every direction. Since $f$ is not eventually annular, this implies the rotation set of any lift of $f$ is singleton containing a totally irrational vector. Hence we can invoke Lemma 5.7 to guarantee that $\mathscr{U}_{F}(V)_{t}$ is annular for every $t \in \mathbb{T}$, and thus, $\mathbb{A} \backslash \overline{\mathscr{U}_{F}(V)}$, has exactly two unbounded connected components; and the theorem is proven in this case.

So, now we can assume that $f$ exhibits unbounded horizontal rotational deviations, i.e. if $\tilde{f}: \mathbb{R}^{2} \bigcirc$ is a lift of $f$, then condition (24) holds.

Reasoning by contradiction, let us suppose there exists a nonempty open connected bounded subset $V \subset \mathbb{T} \times \mathbb{A}$ and $t_{0} \in \mathbb{T}$, such that $V \cap \Omega(F) \neq \varnothing$ and $\mathbb{A} \backslash{\overline{\mathscr{U}_{F}(V)}}_{t_{0}}$ has just one unbounded connected component.

Since $f$ exhibits uniformly bounded vertical deviations, by (45) we know the set $\mathscr{U}_{F}(V)$ is bounded in $\mathbb{T} \times \mathbb{A}$ and so there exists a constant $C_{v}>0$ such that

$$
\mathscr{U}_{F}(V) \subset \mathbb{T} \times\left(\mathbb{T} \times\left(-C_{v}, C_{v}\right)\right) .
$$

Thus, since $\mathbb{A} \backslash{\overline{\mathscr{U}_{F}(V)}}_{t_{0}}$ is open, its unique unbounded connected component is arc-wise connected as well. So there exists a continuous curve $\gamma:[0,1] \rightarrow \mathbb{A}$ such that $\gamma(0) \in \mathbb{T} \times\left(-\infty,-C_{v}-1\right), \gamma(1) \in \mathbb{T} \times\left(C_{v}+1,+\infty\right)$ and $\gamma(s) \notin{\overline{\mathscr{U}_{F}(V)}}_{t_{0}}$, for every $s \in[0,1]$. By compactness of the (image of the) curve $\gamma$ and the set $\overline{\mathscr{U}_{F}(V)}$, there exists $\varepsilon>0$ such that

$$
\gamma(s) \notin{\overline{\mathscr{U}_{F}(V)}}_{t}, \quad \forall t \in B_{\varepsilon}\left(t_{0}\right), \forall s \in[0,1]
$$

where $B_{\mathcal{\varepsilon}}\left(t_{0}\right):=\left\{t \in \mathbb{T}: d_{\mathbb{T}}\left(t, t_{0}\right)<\varepsilon\right\}$. Since the number $\rho$ appearing in the base dynamics of the skew-product $F$ is irrational, this implies that there exists $N \in \mathbb{N}$ such that

$$
\bigcup_{j=0}^{N} T_{\rho}^{j}\left(B_{\varepsilon}\left(t_{0}\right)\right)=\mathbb{T} .
$$

So, for every $t \in \mathbb{T}$ there are $n_{t} \in\{0,1, \ldots, N\}$ and $t^{\prime} \in B_{\varepsilon}\left(t_{0}\right)$ such that $T_{\rho}^{n_{t}}\left(t^{\prime}\right)=t$ and then,

$$
F^{n_{t}}\left(t^{\prime}, \gamma(s)\right) \notin \overline{\mathscr{U}_{F}(V)}, \quad \forall s \in[0,1] .
$$

Then, putting together (53) and (54) we conclude that for every $t \in \mathbb{T}$, every connected component of the set $\mathscr{U}_{F}(V)_{t}$ is lift-bounded in $\mathbb{A}$. Moreover, they are uniformly bounded, i.e. there is a real constant $C>0$ such that $\operatorname{diam} \tilde{U}<C$, for every $t \in \mathbb{T}$ and every connected component $\tilde{U}$ of the open set $\tilde{\pi}^{-1}\left(\mathscr{U}_{F}(V)_{t}\right) \subset \mathbb{R}^{2}$, where $\tilde{\pi}: \mathbb{R}^{2} \rightarrow \mathbb{A}$ denotes the covering map given by (18).

Let us see this leads us to a contradiction. In fact, by Proposition 5.1 we know the set $\Omega(F)$ is $\Gamma$-invariant, and we are assuming $V \cap \Omega(F) \neq \varnothing$. So, there exists $s \in \mathbb{R}$ such that $\Gamma^{\mathcal{S}}(V) \cap \Omega(F) \cap(\{0\} \times \mathbb{A}) \neq \varnothing$. Let us consider the fiber of $\Gamma^{\mathcal{S}}(V)$ over the point $0 \in \mathbb{T}$, i.e. the set $\left(\Gamma^{\mathcal{S}}(V)\right)_{0}$. Since $f$ is $\Omega$-recurrent, by Theorem 5.5 we know that

$$
\left(\Gamma^{S}(V)\right)_{0} \cap \hat{\pi}^{-1}(\Omega(f)) \neq \varnothing
$$


Hence, there is a connected component $\tilde{V}$ of the open set $\tilde{\pi}^{-1}\left(\left(\Gamma^{s}(V)\right)_{0}\right) \subset \mathbb{R}^{2}$ such that $\pi(\tilde{V}) \cap \Omega(f) \neq \varnothing$, where $\tilde{\pi}: \mathbb{R}^{2} \rightarrow \mathbb{A}$ and $\pi: \mathbb{R}^{2} \rightarrow \mathbb{T}^{2}$ are the natural covering maps.

Now notice, since we are assuming $f$ is periodic point free and non-eventually annular, by Proposition 2.11, every non-wandering point is fully essential, and recalling $f$ exhibits unbounded horizontal rotational deviations (i.e. condition (24) holds), we can invoke Lemma 2.13 to conclude that

$$
\sup _{n \in \mathbb{Z}} \operatorname{diam}\left(\operatorname{pr}_{1}\left(\tilde{f}^{n}(\tilde{V})\right)\right)=\infty,
$$

Finally, by (45) we know that (55) contradicts the fact that every connected components of ${\overline{\mathscr{U}_{F}(V)}}_{t}$ are uniformly lift-bounded, for each $t \in \mathbb{T}$. Thus, we have proved that $\mathbb{A} \backslash \overline{\mathscr{U}_{F}(V)_{t}}$ has exactly two unbounded components for every $t \in \mathbb{T}$.

In order to finish the proof of Theorem 5.6, it just remains to show that the set $\mathscr{U}_{F}(V)$ is $F$-invariant. To do this, by the very definition of $\mathscr{U}_{F}(V)$ (given by (9)), we know that $\mathscr{U}_{F}(V)$ is a periodic set and $F\left(\mathscr{U}_{F}(V)\right) \cap \mathscr{U}_{F}(V) \neq \varnothing$ if and only if $F\left(\mathscr{U}_{F}(V)\right)=\mathscr{U}_{F}(V)$.

So, reasoning by contradiction, if we suppose $\mathscr{U}_{F}(V)$ is not $F$-invariant, then we get that $F\left(\mathscr{U}_{F}(V)\right)$ and $\mathscr{U}_{F}(V)$ are disjoint. We have already proved that the complement of the closed set $\overline{\mathscr{U}_{F}(V)_{t}}$ has exactly two unbounded components on the annulus $\mathbb{A}$, for every $t \in \mathbb{T}$, which are called the upper and lower components and are denoted by $U^{+}\left({\overline{\mathscr{U}_{F}(V)_{t}}}_{t}\right)$ and $U^{-}\left({\overline{\mathscr{U}_{F}(V)}}_{t}\right)$, respectively. So, if $\mathscr{U}_{F}(V)$ is not invariant by $F$, since $\overline{\mathscr{U}_{F}(V)}$ is connected then we have either $F\left(\mathscr{U}_{F}(V)_{t}\right) \subset$ $U^{+}\left({\overline{\mathscr{U}_{F}(V)}}_{T_{\rho}(t)}\right)$ or $F\left(\mathscr{U}_{F}(V)_{t}\right) \subset U^{-}\left({\overline{\mathscr{U}_{F}(V)}}_{T_{\rho}(t)}\right)$, for every $t \in \mathbb{T}$. Without loss of generality, if we assume the first inclusion holds for every $t \in \mathbb{T}$, we conclude that

$$
F\left(\overline{U^{+}\left({\overline{\mathscr{U}_{F}(V)_{t}}}_{t}\right)}\right) \subset U^{+}\left({\overline{\mathscr{U}_{F}(V)}}_{T_{\rho}(t)}\right), \quad \forall t \in \mathbb{T} .
$$

This implies the open set $U^{+}$given by

$$
U^{+}:=\bigcup_{t \in \mathbb{T}}\{t\} \times U^{+}\left({\overline{\mathscr{U}_{F}(V)}}_{t}\right) \subset \mathbb{T} \times \mathbb{A},
$$

satisfies $F\left(\overline{U^{+}}\right) \subset U^{+}$and this clearly contradicts the fact there exists $n>1$ such that $F^{n}\left(\mathscr{U}_{F}(V)\right)=\mathscr{U}_{F}(V)$.

\section{Proof of Theorem A}

In order to show that estimate (2) is a necessary condition for the existence of an irrational circle factor, no assumption about the non-wandering set is required. In fact, the proof is based in rather classical arguments and all the details can be found for instance in [JT17, Lemma 3.1]. Let us just mention that in this case the irrationality of $\rho$ follows from the non-annularity hypothesis.

To finish the proof of Theorem A, from now on let us suppose $\tilde{f}$ is a lift of $f$ and satisfies condition (2). By Proposition 2.4 and the remark about rotational deviations at the beginning of $\$ 2.6$, there is no loss of generality assuming $f \in$ Homeo $_{k}\left(\mathbb{T}^{2}\right)$, for some $k \in \mathbb{Z}$, and $v=(0,1)$ in estimate (2). So, the lift $\tilde{f}: \mathbb{R}^{2} ๖$ of $f$ commutes with the horizontal translation $T_{(1,0)}: \mathbb{R}^{2} ๖$ and, consequently, induces an annulus homeomorphism $\hat{f}: \mathbb{A} \bigcirc$ characterized by the semi-conjugacy equation $\tilde{\pi} \circ \tilde{f}=\hat{f} \circ \tilde{\pi}$.

Now, we want to construct a surjective continuous map $h: \mathbb{T}^{2} \rightarrow \mathbb{T}$ such that $h \circ f=T_{\rho} \circ h$, where $\rho$ is the irrational number given by (2). By [JP15, Theorem 1] we know that there is no loss of generality assuming that each fiber $h^{-1}(y)$, with 
$y \in \mathbb{T}$, is an annular continuum as defined in $§ 2.4 .2$. So, following Jäger [Jäg09], we will define the semi-conjugacy $h$ starting from its family of fibers.

More precisely, due to technical reasons we will start working on the annulus $\mathbb{A}$ instead of $\mathbb{T}^{2}$ and we will construct a family of continua $\left\{\mathscr{C}^{s} \subset \mathbb{A}: s \in \mathbb{R}\right\}$ such that each $\mathscr{C}^{s}$ is an essential annular continuum in $\mathbb{A}$, they are well ordered according to the index, i.e.

$$
\mathscr{C}^{s} \subset U^{-}\left(\mathscr{C}^{r}\right), \text { for all } s<r,
$$

where $U^{-}(\cdot)$ denotes the lower connected component of the complement in $\mathbb{A}$ of the corresponding essential annular continuum as defined in $\$ 2.4 .2$; and the family satisfies the following equivariant properties:

$$
\begin{aligned}
\hat{T}_{1}\left(\mathscr{C}^{s}\right) & =\mathscr{C}^{s+1}, \\
\hat{f}\left(\mathscr{C}^{s}\right) & =\mathscr{C}^{s+\rho}, \quad \forall s \in \mathbb{R} .
\end{aligned}
$$

Observe that, in particular, (56) implies that the continua are two-by-two disjoint, i.e. $\mathscr{C}^{s} \cap \mathscr{C}^{r}=\varnothing$, whenever $r \neq s$.

Then, we define the map $\hat{h}: \mathbb{A} \rightarrow \mathbb{R}$ by

$$
\hat{h}(z):=\inf \left\{s \in \mathbb{R}: z \in U^{-}\left(\mathscr{C}^{s}\right)\right\}, \quad \forall z \in \mathbb{A} .
$$

By (57) we know that $\hat{h} \circ \hat{T}_{1}=T_{1} \circ \hat{h}$ and, by (58), $\hat{h} \circ \hat{f}=T_{\rho} \circ \hat{h}$. Thus, $\hat{h}$ is the lift of a map $h: \mathbb{T}^{2} \rightarrow \mathbb{T}$ and it clearly holds $h \circ f=T_{\rho} \circ h$. So, in order to show that $h$ is indeed a semi-conjugacy, it just remains to prove that $\hat{h}$, and then $h$ as well, is continuous. This is a consequence of the irrationality of $\rho$ and the fact that the essential annular continua $\left\{\mathscr{C}^{s}: s \in \mathbb{R}\right\}$ are two-by-two disjoint. The reader can find more details about the proof of the continuity of $\hat{h}$ given by (59), assuming (56), (57) and (58), in either [Jäg09, page 615] or [JP15, Lemma 3.2].

6.1. The construction of continua $\mathscr{C}^{s}$. In order to finish the proof of Theorem A, it remains to construct the family of essential annular continua $\left\{\mathscr{C}^{s}: s \in \mathbb{R}\right\}$ satisfying properties (56), (57) and (58).

To do that, let $F: \mathbb{T} \times \mathbb{A} \bigcirc$ be the $\rho$-centralized skew-product induced by $\tilde{f}$ given by (44) and where $\rho$ is the irrational number appearing in (2). Since $f$ exhibits small wandering domains (Definition 2.3) and is periodic point free, by Proposition 2.8 we know that $f$ is $\Omega$-recurrent, and hence, by Theorem 5.5 we know that $z \in \Omega(f)$ if and only $(0, \hat{z}) \in \Omega(F)$, for every $\hat{z} \in \hat{\pi}^{-1}(z)$.

The main new idea of the proof of Theorem $\mathrm{A}$ is given by the following lemma, which could seem at first glance to be rather technical, but is the core of the method:

Lemma 6.1. There exists an open bounded connected F-invariant subset $\mathscr{T} \subset$ $\mathbb{T} \times \mathbb{A}$ such that

$$
\left(\mathscr{T} \backslash \Gamma^{-s}(\overline{\mathscr{T}})\right) \cap \Omega(F) \neq \varnothing, \quad \forall s \in \mathbb{R} \backslash\{0\} .
$$

Before proving Lemma 6.1, let us see how the open set $\mathscr{T}$ can be used to construct our family of annular continua $\left\{\mathscr{C}^{s}: s \in \mathbb{R}\right\}$.

First, observe that since $\mathscr{T}$ is bounded, connected and F-invariant, and intersects $\Omega(F)$, it holds $\mathscr{T}=\mathscr{U}_{F}(\mathscr{T})$. So, by Theorem 5.6 we know the set $\mathbb{A} \backslash \Gamma^{-s}(\overline{\mathscr{T}})_{t}$ has exactly two unbounded connected components, for every $t \in \mathbb{T}$ and any $s \in \mathbb{R}$. Hence, we can define the sets

$$
\mathscr{T}_{t}^{s,-}:=U^{-}\left(\Gamma^{-s}(\overline{\mathscr{T}})_{t}\right) \subset \mathbb{A}, \quad \forall t \in \mathbb{T}, \forall s \in \mathbb{R},
$$

i.e. it is the lower unbounded connected component of the set $\mathbb{A} \backslash \Gamma^{-s}(\overline{\mathscr{T}})_{t}$. Then we write

$$
\mathscr{C}^{s}:=\partial_{\mathbb{A}}\left(\mathscr{T}_{0}^{s,-}\right) \subset \mathbb{A}, \quad \forall s \in \mathbb{R} .
$$


Notice that each $\mathscr{C}^{s}$ is an essential annular continuum. Moreover, since $F$ commutes with $\Gamma^{-1}$ and $\Gamma^{-1}(t, z)=(t, z+1)$, for all $(t, z) \in \mathbb{T} \times \mathbb{A}$, then (57) clearly holds.

In order to prove (58), observe that, since $\Gamma^{-s}(\mathscr{T})$ is $F$-invariant and $F$ preserves the ends of the space $\mathbb{T} \times \mathbb{A}$, we get

$$
F\left(\{t\} \times \mathscr{T}_{t}^{s,-}\right)=\left\{T_{\rho}(t)\right\} \times \mathscr{T}_{T_{\rho}(t)}^{s,-}, \quad \forall t \in \mathbb{T}, \forall s \in \mathbb{R},
$$

and on the other hand,

$$
\Gamma^{-\rho}\left(\left\{T_{\rho}(t)\right\} \times \mathscr{T}_{T_{\rho}(t)}^{s,-}\right)=\{t\} \times \mathscr{T}_{t}^{s+\rho,-}, \quad \forall t \in \mathbb{T}, \forall s \in \mathbb{R} .
$$

Putting together (45), (62) and (63) we get

$$
\hat{f}\left(\mathscr{C}^{s}\right)=\mathscr{C}^{s+\rho}, \quad \forall s \in \mathbb{R},
$$

and (58) is proven.

So, it remains to prove that the continua $\left\{\mathscr{C}^{s}: s \in \mathbb{R}\right\}$ given by (61) satisfies condition (56). To do that, it is important to notice that is enough to show that

$$
\mathscr{C}^{s} \cap \mathscr{C}^{r}=\varnothing, \quad \forall r, s \in \mathbb{R}, s \neq r .
$$

In fact, one can easily check that condition (56) follows from (64) and the combinatorics of these essential annular continua given by conditions (57), (58) and the fact that

$$
\frac{\operatorname{pr}_{2}\left(\hat{f}^{n}-i d\right)}{n} \rightarrow \rho, \quad \text { as } n \rightarrow \infty
$$

uniformly on $\mathbb{A}$, which is a direct consequence of (2).

So, condition (64) is the only remaining step to prove that Theorem A is consequence of Lemma 6.1. To do that, let $r$ be an arbitrary real number and $s \in(0,1)$. We will show that $\mathscr{C}^{r}$ and $\mathscr{C}^{r+s}$ are disjoint. Then, recalling that $\mathscr{C}^{r}$ and $\mathscr{C}^{r+s}$ are the boundary of the open set $\mathscr{T}_{0}^{r,-}$ and $\mathscr{T}_{0}^{r+s,-}$, respectively, we consider the open set

$$
\check{V}:=\Gamma^{-r}(\mathscr{T}) \backslash \Gamma^{-(r+s)}(\overline{\mathscr{T}})=\Gamma^{-r}\left(\mathscr{T} \backslash \Gamma^{-s}(\overline{\mathscr{T}})\right) \subset \mathbb{T} \times \mathbb{A} .
$$

Since $\mathscr{T}$ and $\Omega(F)$ are $F$-invariant, and by Proposition $5.1 F$ and $\Gamma$ commute, we get that $\check{V}$ is $F$-invariant, and by Lemma 6.1 , it holds $\check{V} \cap \Omega(F) \neq \varnothing$. Moreover, we claim there exists a connected component $V \in \pi_{0}(\check{V})$ such that

$$
V \cap \Omega(F) \cap(\{0\} \times \mathbb{A}) \neq \varnothing .
$$

To prove this, first let $V^{\prime}$ be any connected component of $\check{V}$ that intersects $\Omega(F)$. If $(t, \hat{z})$ is an arbitrary point of $V^{\prime} \cap \Omega(F)$, since $F$ and $\Gamma$ commute and $V^{\prime}$ is open, there is a positive number $\varepsilon>0$ such that

$$
\Gamma^{u}(t, \hat{z}) \in V^{\prime} \cap \Omega(F) \cap\{t+\pi(u)\} \times \mathbb{A}, \quad \forall u \in(-\varepsilon, \varepsilon) .
$$

Then, since $\rho$ is irrational, there is $n \in \mathbb{Z}$ such that $0 \in T_{\rho}^{n}\left(B_{\varepsilon}(t)\right) \subset \mathbb{T}$; and hence, $V:=F^{n}\left(V^{\prime}\right) \in \pi_{0}(\check{V})$ satisfies $(65)$.

Now, recalling we are assuming $f$ is not eventually annular, there are two possible cases to be considered: either $f$ is a totally irrational pseudo-rotation exhibiting uniformly bounded rotational deviations (i.e. estimate (27) holds); or $f$ exhibits uniformly bounded vertical rotational deviations and unbounded horizontal deviations (i.e. estimate (24) holds).

In the first case, i.e. when $f$ is a totally irrational pseudo-rotation and (27) holds, the disjointness of continua $\mathscr{C}^{r}$ and $\mathscr{C}^{r+s}$ easily follows from Lemma 5.7. In fact, $V$ is a connected component of an $F$-invariant set and intersects $\Omega(F)$. So, $V=\mathscr{U}_{F}(V)$ and by Lemma 5.7 we know that $V_{t}$ is annular, for every $t \in \mathbb{T}$. This implies $V_{t}$ separates $\mathscr{C}^{r}$ and $\mathscr{C}^{r+s}$. 
The second case, i.e. when $f$ exhibits unbounded horizontal deviations, will follow as consequence of Lemma 2.14, but it is a little more involved.

Let $(0, \hat{z})$ be any point belonging to $V \cap \Omega(F)$, and $\delta>0$ be a sufficiently small number such that

$$
B:=\left(B_{\delta}(0, \hat{z})\right)^{\delta, 0}=\bigcup_{|u|<\delta} \Gamma^{u}\left(B_{\delta}(0, \hat{z})\right) \subset V .
$$

Without loss of generality we can assume $\delta<1 / 4$. We will show that there exists $i \in \mathbb{N}$ and $q \in \mathbb{Z}$ such that

$$
\left(\Gamma^{-q}(B) \cap F^{i}(B)\right)_{0} \subset \mathbb{A}
$$

is an essential set, and this will prove that $\mathscr{C}^{r}$ and $\mathscr{C}^{r+s}$ are disjoint. In fact, we know that $B_{0} \cap \mathscr{C}^{r} \subset V_{0} \cap \mathscr{C}^{r}=\varnothing$, and hence, by $(57),\left(\Gamma^{-q}(B)\right)_{0} \cap \hat{T}_{q}\left(\mathscr{C}^{r}\right)=$ $\hat{T}_{q}\left(B_{0}\right) \cap \mathscr{C}^{r+q}=\varnothing$, where $B_{0}$ denotes the fiber of $B \subset \mathbb{T} \times \mathbb{A}$ over the point $0 \in \mathbb{T}$. Analogously, one can show that $\left(\Gamma^{-q}(B)\right)_{0} \cap \mathscr{C}^{r+s+q}=\varnothing$; and since we are assuming $0<s<1$, we know that $B_{0} \cap \mathscr{C}^{r+q}=B_{0} \cap \mathscr{C}^{r+s+q}=\varnothing$, for any $q \in \mathbb{Z}$. So, condition (67) clearly shows that $\mathscr{C}^{r+q} \cap \mathscr{C}^{r+s+q}=\varnothing$, and by (57), this holds if and only if $\mathscr{C}^{r} \cap \mathscr{C}^{r+s}=\varnothing$.

So, let us show (67) holds. By the minimality of $T_{\rho}: \mathbb{T} \wp$, we know there exists a natural number $N$ such that for any $m \in \mathbb{Z}$, there is $i \in\{m, m+1, \ldots, m+N\}$ such that $T_{\rho}^{i}(0) \in B_{\delta / 4}(0) \subset \mathbb{T}$.

On the other hand, if we write $z:=\hat{\pi}(\hat{z})$, by Theorem 5.5 we know that $z \in \Omega(f)$. Let $\tilde{z}$ be any point in $\pi^{-1}(z)=\tilde{\pi}^{-1}(\hat{z})$. Applying Lemma 2.14 for the open set $B_{\delta / 4}(\tilde{z}) \subset \mathbb{R}^{2}$ and the natural number $N$ given by the above condition, we know that there is $m \in \mathbb{N}$ such that for every $j \in\{m, m+1, \ldots, m+N\}$, there are $p_{j}, p_{j}^{\prime}, q_{j} \in \mathbb{Z}$ with $p_{j} \neq p_{j}^{\prime}$ and such that $\tilde{f}^{j}\left(B_{\delta / 4}(\tilde{z})\right) \cap T_{\left(p_{j}, q_{j}\right)}\left(B_{\delta / 4}(\tilde{z})\right) \neq \varnothing$ and $\tilde{f}^{j}\left(B_{\delta / 4}(\tilde{z})\right) \cap T_{\left(p_{j}^{\prime}, q_{j}\right)}\left(B_{\delta / 4}(\tilde{z})\right) \neq \varnothing$; or in other words, we get that the open set $\hat{T}_{q_{j}}\left(\tilde{\pi}\left(B_{\delta / 4}(\tilde{z})\right)\right) \cup \hat{f}^{j}\left(\tilde{\pi}\left(B_{\delta / 4}(\tilde{z})\right)\right)$ is essential in $\mathbb{A}$, for every $j \in\{m, \ldots, m+N\}$.

Then, let $i$ be a natural number such that $i \in\{m, \ldots, m+N\}$ and $T_{\rho}^{i}(0) \in B_{\delta / 4}(0)$, and let us consider the open set

$$
\hat{B}^{(i)}:=\hat{T}_{q_{i}}\left(\tilde{\pi}\left(B_{\delta / 4}(\tilde{z})\right)\right) \cup \hat{f}^{i}\left(\tilde{\pi}\left(B_{\delta / 4}(\tilde{z})\right)\right)=\hat{T}_{q_{i}}\left(B_{\delta / 4}(\hat{z})\right) \cup \hat{f}^{i}\left(B_{\delta / 4}(\hat{z})\right) \subset \mathbb{A},
$$

since we are assuming $\delta<1 / 4$. We know that $\hat{B}^{(i)}$ is an essential set and putting together this with Propositions 5.1 and 5.2, and (66), we conclude that there is an integer number $q$ such that the set given by (67) is an essential set in $\mathbb{A}$, as we wanted to prove. Then, (56) is proven.

So, the last step of the proof of Theorem A is

Proof of Lemma 6.1. The proof of this lemma is considerably simpler in the case that $\Omega(f)$ has nonempty interior (e.g. when $f$ is non-wandering) than in the case where the wandering set is dense in $\mathbb{T}^{2}$.

So, for the sake of simplicity of the exposition let us start proving the lemma under the assumption that the non-wandering set has nonempty interior. In such a case, let $V \subset \Omega(f)$ be an open, nonempty, lift-bounded and connected set.

Since we are assuming $f$ is periodic point free and exhibits small wandering domains, by Proposition 2.8 we know that $f$ is $\Omega$-recurrent, and thus, by Theorem 5.5 it holds $\{0\} \times \hat{\pi}^{-1}(V) \subset \Omega(F) \subset \mathbb{T} \times \mathbb{A}$, where $\hat{\pi}: \mathbb{A} \rightarrow \mathbb{T}^{2}$ is the natural covering map given by (18). 
Let $\hat{V}$ be any connected component of $\hat{\pi}^{-1}(V) \subset \mathbb{A}$. Since we are assuming $V$ is lift-bounded, $\hat{V}$ is bounded in $\mathbb{A}$ as well. Then, we define

$$
\mathscr{T}:=\mathscr{U}_{F}\left(\hat{V}^{\frac{1}{2}, 0}\right)
$$

where $\hat{V}^{\frac{1}{2}, 0}$ denotes the $1 / 2$-block induced by $V$ and centered at $0 \in \mathbb{T}$ given by $(47)$, and $\mathscr{U}_{F}(\cdot)$ is given by (9). By Theorem 5.6 we know that $\mathscr{T}$ is F-invariant, and by Proposition 5.1 we know that $\mathscr{T} \subset \Omega(F)$.

On the other hand, since $\hat{V}$ is bounded in $\mathbb{A}$, by Proposition $5.4 \mathscr{T}$ is bounded in $\mathbb{T} \times \mathbb{A}$ as well. This implies that for every $s \neq 0, \mathscr{T} \backslash \Gamma^{-s}(\overline{\mathscr{T}})$ is nonempty and, clearly $\mathscr{T} \backslash \Gamma^{-s}(\overline{\mathscr{T}}) \subset \Omega(F)$. Hence, Lemma 6.1 is proven under the additional hypothesis that $\Omega(f)$ has nonempty interior.

So, from now on let us suppose the non-wandering set $\Omega(f)$ has empty interior.

Let $z_{0}$ be any point of $\Omega(f)$ and $\hat{z}_{0}$ an arbitrary point in $\hat{\pi}^{-1}\left(z_{0}\right) \subset \mathbb{A}$. Now let us define the set $\hat{V}$ as the wandering completion of the open unit ball $B_{1}\left(\hat{z}_{0}\right) \subset \mathbb{A}$, i.e. $\hat{V}$ is given by

$$
\hat{V}:=B_{1}\left(\hat{z}_{0}\right) \cup \bigcup\left\{\hat{\pi}^{-1}(W): W \in \pi_{0}\left(\mathbb{T}^{2} \backslash \Omega(f)\right), \hat{\pi}^{-1}(W) \cap B_{1}\left(\hat{z}_{0}\right) \neq \varnothing\right\} .
$$

Since $f$ exhibits small wandering domains, $\hat{V}$ is open, bounded and connected, and since $z_{0} \in \Omega(f)$, by Theorem 5.5 we know $\left(0, \hat{z}_{0}\right) \in \Omega(F) \cap \mathscr{T}$. Hence, the set $\mathscr{T}:=\mathscr{U}_{F}\left(\hat{V}^{\frac{1}{2}}, 0\right)$ is again $F$-invariant, open and bounded in $\mathbb{T} \times \mathbb{A}$. This implies $\mathscr{T} \backslash \Gamma^{-s}(\overline{\mathscr{T}}) \neq \varnothing$, for every $s \in \mathbb{R} \backslash\{0\}$.

So, it just remain to prove that the set $\mathscr{T} \backslash \Gamma^{-s}(\overline{\mathscr{T}})$ intersects $\Omega(F)$, for every $s \neq 0$. To do that, let us just consider the case where $s>0$; the other one is completely analogous.

Now, observe that combining Proposition 2.7, Proposition 5.2 and (69) we know that each connected component of the open set $\mathscr{T} \cap \mathscr{W}(F)$ is a $1 / 2$-block, each of them is a wandering set for $F$ and their $\Gamma$-orbits are two-by-two disjoint. More precisely, if $\check{W}_{1}, \check{W}_{2}, \ldots$ denote the (infinitely many) connected components of $\mathscr{T} \cap$ $\mathscr{W}(F)$, then for each $n \geqslant 1$ there exists a unique connected component $W_{n}$ of $\mathscr{W}(f)$ such that if we choose any connected component $\hat{W}_{n}$ of the open set $\hat{\pi}^{-1}\left(W_{n}\right) \subset \mathbb{A}$, there is a unique $r_{n} \in \mathbb{R}$ satisfying

$$
\check{W}_{n}=\bigcup_{|u|<\frac{1}{2}} \Gamma^{u+r_{n}}\left(\{0\} \times \hat{W}_{n}\right) .
$$

On the other hand, by our definition (69) and Theorem 5.5 again, we see that for each $n \geqslant 1$, there exists at least a point $\hat{z}_{n} \in \partial_{\mathbb{A}}\left(\hat{W}_{n}\right)$ such that

$$
\Gamma^{r_{n}+u}\left(0, \hat{z}_{n}\right) \in \overline{W_{n}} \cap \mathscr{T} \cap \Omega(F), \quad \forall u \in(-1 / 2,1 / 2) .
$$

Reasoning by contradiction let us suppose that there exists $s>0$ such that the open set $D:=\mathscr{T} \backslash \Gamma^{-s}(\overline{\mathscr{T}})$ does not intersects $\Omega(F)$. This implies, invoking (70), that $D$ has infinitely many connected components and all of them can be enumerated as follows:

$$
D_{n}:=\bigcup_{|u|<\frac{s}{2}} \Gamma^{r_{n}+\frac{1-s}{2}+u}\left(\{0\} \times \hat{W}_{n}\right), \quad \forall n \geqslant 1,
$$

and $D=\bigsqcup_{n \geqslant 1} D_{n}$

Then, we will construct a subsequence $\left(D_{n_{j}}\right)_{j \geqslant 1}$ of connected components of $D$ such that for every $M>0$ there is a $j \geqslant 1$ with $D_{n_{j}} \cap \mathbb{T} \times(-\infty,-M) \neq \varnothing$, contradicting the fact that $D$ was a bounded subset of $\mathbb{T} \times \mathbb{A}$. 
To do that, we will define the sequence $\left(D_{n_{j}}\right)_{j \geqslant 1}$ inductively. Let us start defining $n_{1}=1$. By $(71)$ we know that

$$
w_{1}:=\Gamma^{r_{1}+\frac{2-s}{4}}\left(0, \hat{z}_{1}\right) \in \overline{D_{1}} \cap \mathscr{T} \cap \Omega(F),
$$

but since $D$ and $\Omega(F)$ are disjoint, we get that $w_{1} \notin D_{n_{1}}=D_{1}$. So, $w_{1} \in \Gamma^{-s}(\overline{\mathscr{T}})$. That means that there exists $n_{2} \in \mathbb{N}$ such that

$$
d_{\mathbb{T} \times \mathbb{A}}\left(\Gamma^{\mathcal{S}}\left(w_{1}\right), \check{W}_{n_{2}}\right)<\frac{1}{2^{2}},
$$

and we define

$$
w_{2}:=\Gamma^{r_{n_{2}}+\frac{2-s}{4}}\left(0, \hat{z}_{n_{2}}\right) \in \bar{D}_{n_{2}} \cap \mathscr{T} \cap \Omega(F) .
$$

Inductively, supposing that $n_{k} \in \mathbb{N}$ and $w_{k} \in \mathbb{T} \times \mathbb{A}$ has been already defined, we choose $n_{k+1} \in \mathbb{N}$ as any natural number satisfying

$$
d_{\mathbb{T} \times \mathbb{A}}\left(\Gamma^{s}\left(w_{k}\right), \check{W}_{n_{k+1}}\right)<\frac{1}{2^{k+1}},
$$

and then we define

$$
w_{j+1}:=\Gamma^{r_{n_{k+1}}+\frac{2-s}{4}}\left(0, \hat{z}_{n_{k+1}}\right) \in \bar{D}_{n_{k+1}} \cap \mathscr{T} \cap \Omega(F) .
$$

Then, putting together (70), (71), (72), (73) and (74) we conclude that

$$
\operatorname{pr}_{3}\left(w_{k+1}\right) \leqslant \operatorname{pr}_{3}\left(w_{k}\right)-s+\operatorname{diam} W_{n_{k+1}}+\frac{1}{2^{k}}, \quad \forall k \geqslant 1 .
$$

Iterating this last estimate one easily gets

$$
\begin{aligned}
\operatorname{pr}_{3}\left(w_{k+1}\right) & \leqslant \operatorname{pr}_{3}\left(w_{1}\right)+\sum_{j=1}^{k}\left(\operatorname{diam} W_{n_{j+1}}-s\right)+\sum_{j=1}^{k} \frac{1}{2^{j}} \\
& <\operatorname{pr}_{3}\left(w_{1}\right)+1+\sum_{j=1}^{k}\left(\operatorname{diam} W_{n_{j+1}}-s\right) \rightarrow-\infty,
\end{aligned}
$$

as $k \rightarrow+\infty$, where the last limit follows from the small wandering domain hypothesis which implies diam $W_{n_{k}} \rightarrow 0$, as $n_{k} \rightarrow+\infty$.

Since $w_{k} \in \bar{D}_{n_{k}} \subset \bar{D}$, for every $k \in \mathbb{N}$, estimate (75) clearly contradicts the fact that $D$ was bounded in $\mathbb{T} \times \mathbb{A}$.

\section{REFERENCES}

[AZ05] S. Addas-Zanata, On properties of the vertical rotation interval for twist mappings, Ergodic Theory and Dynamical Systems 25 (2005), no. 03, 641-660.

[DM97] E. Doeff and M. Misiurewicz, Shear rotation numbers, Nonlinearity 10 (1997), no. 6, $1755-1762$.

[Doe97] E. Doeff, Rotation measures for homeomorphisms of the torus homotopic to a Dehn twist, Ergodic Theory Dynam. Systems 17 (1997), no. 3, 575-591.

[Ede19] N. Edeko, On equicontinuous factors of flows on locally path-connected compact spaces, arXiv preprint arXiv:1904.12203, 2019.

[Fat87] A. Fathi, An orbit closing proof of Brouwer's lemma on translation arcs, Enseign. Math. (2) 33 (1987), no. 3-4, 315-322.

[FM90] J. Franks and M. Misiurewicz, Rotation sets of toral flows, Proc. Amer. Math. Soc. 109 (1990), no. 1, 243-249.

[GH55] W. Gottschalk and G. Hedlund, Topological dynamics, vol. 36, American Mathematical Soc., 1955.

[HJ17] T. Hauser and T. Jäger, Monotonicity of maximal equicontinuous factors and an application to toral flows, ArXiv preprint 1711.05672. To appear in Proceedings of the American Mathematical Society, 2017.

[Jäg09] T. Jäger, Linearization of conservative toral homeomorphisms, Inventiones Mathematicae 176 (2009), no. 3, 601-616. 
[JP15] T. Jäger and A. Passeggi, On torus homeomorphisms semiconjugate to irrational rotations, Ergodic Theory and Dynamical Systems 35 (2015), no. 07, 2114-2137.

[JT17] T. Jäger and F. Tal, Irrational rotation factors for conservative torus homeomorphisms, Ergodic Theory and Dynamical Systems 37 (2017), no. 5, 1537-1546.

[KK09] A. Kocsard and A. Koropecki, A mixing-like property and inexistence of invariant foliations for minimal diffeomorphisms of the 2-torus, Proceedings of the American Mathematical Society 137 (2009), no. 10, 3379-3386.

[Koc16] A. Kocsard, On the dynamics of minimal homeomorphisms of $\mathbb{T}^{2}$ which are not pseudorotations, ArXiv:1611.03784. To appear in Annales scientifiques de l'Ecole normale supérieure, 2016.

[KPR18] A. Kocsard and F. Pereira-Rodrigues, Rotational deviations and invariant pseudofoliations for periodic point free torus homeomorphisms, Math. Z. 290 (2018), no. 3-4, 1223-1247.

[KPS16] A. Koropecki, A. Passeggi, and M. Sambarino, The Franks-Misiurewicz conjecture for extensions of irrational rotations, preprint arXiv:1611.05498, 2016.

[KT14] A. Koropecki and F. Tal, Strictly toral dynamics, Inventiones Mathematicae 196 (2014), no. 2, 339-381.

[MZ89] M. Misiurewicz and K. Ziemian, Rotation sets for maps of tori, Journal of the London Mathematical Society 2 (1989), no. 3, 490-506.

[Poi80] H. Poincaré, Mémoire sur les courbes définies par les équations différentielles I-VI, oeuvre I, Gauthier-Villar: Paris (1880), 375-422.

[PS18] A. Passeggi and M. Sambarino, Deviations in the Franks-Misiurewicz conjecture, To appear in Ergodic Theory \& Dynamical Systems, 2018.

[Tao09] T. Tao, Poincaré's legacies: pages from year two of a mathematical blog, American Mathematical Soiety, 2009.

[WZ18] J. Wang and Z. Zhang, The rigidity of pseudo-rotations on the two-torus and a question of Norton-Sullivan, Geom. Funct. Anal. 28 (2018), no. 5, 1487-1516.

E-mail address: akocsard@id.uff.br

ime - Universidade Federal Fluminense. Rua Prof. Marcos Waldemar de Freitas Reis, S/N. Bloco H, $4^{\circ}$ andar. 24.210-201, Gragoatá, Niterói, RJ, Brasil 